\title{
A dynamic network model for the action of low salinity on two-phase flow.
}

\author{
Z. M. Wilmott ${ }^{\mathrm{a}}$, C. J. W. Breward ${ }^{\mathrm{a}}$, S. J. Chapman ${ }^{\mathrm{a}}$ \\ ${ }^{a}$ Mathematical Institute, University of Oxford, Radcliffe Observatory Quarter, Woodstock Road, Oxford OX2 6GG, \\ $U K$
}

\begin{abstract}
Experimental evidence shows that decreasing the salinity of the injection water during the oil recovery process can lead to an increase in the amount of oil recovered. While the ion-exchange reactions which cause this effect are well understood in an industrial setting, there is a limited understanding of how to quantitatively describe the macroscale low salinity effect in terms of the microscale mechanisms. In this paper, we derive a dynamic network model for the salinity-dependent twophase flow of oil and water through a porous medium in which the salinity of the water affects the thickness of the thin water layer separating the oil phase from the solid surface through the multicomponent ionic exchange mechanism, which results in a salinity-dependent slip condition on the effective oil-solid interface. We solve the network model numerically for a drainage stage followed by waterflood stage on a $30 \times 30$ network with random pore and throat radii distributions, and present results averaged over multiple simulations. Low-salinity waterflooding is compared with high-salinity waterflooding in both secondary and tertiary mode. Our model is able to reproduce the low salinity effect observed experimentally, in which the amount of oil produced increases as the salinity of the injection brine decreases.
\end{abstract}

Keywords: Multiphase flow, Flow in porous media, Network modelling, Low salinity, Oil recovery

\section{Introduction}

The oil recovery process typically involves the injection of fluid into an oil reservoir via a number of injection wells. This increases the pressure within the reservoir in order to mobilise oil trapped within the pores of the reservoir rock. However, a large percentage of the oil often remains undisturbed and cannot be retrieved using standard injection methods alone. In order to maximise recovery, a number of enhanced recovery techniques have been developed which enable the release of additional oil. One such method is the injection of low salinity water as either a secondary or tertiary recovery process.

While the observation that reducing the salinity of the injection water can lead to a greater recovery is not new (Buckley et al., 1989; Jadhunandan, 1990; Jadhunandan and Morrow, 1991, 1995), there is still some debate over the dominant microscale mechanism which causes the low salinity effect. Tang and Morrow (1999) propose that small particles of clay detach from the

Email addresses: wilmott@maths.ox.ac.uk (Z. M. Wilmott), breward@maths.ox.ac.uk (C. J. W. Breward), chapman@maths.ox.ac.uk (S. J. Chapman) 
surface of the reservoir rock due to a reduction in the strength of the attractive colloidal forces in the low-salinity regime. These clay particles, known as 'fines', act as a surfactant between the oil and water phases, which improves the oil-in-water emulsion that forms in the reservoir and consequently aids the recovery process. Furthermore, these fines are transported with the fluid and can block the pores that have been flushed of oil. This can lead to an increase in the pressure, which forces the water to find new paths through the pores. Hence, oil that had previously been missed can be recovered. Alternatively, a number of authors have suggested that a decrease in the salinity leads to the build up of a viscoelastic interface between the oil and the water (Alvarado et al., 2014; Moradi et al., 2010; Wang and Alvarado, 2012). This acts to stabilise the water-in-oil emulsion in a reservoir, which leads to a greater recovery. In contrast, Austad et al. (2010) attribute the observed increase in the amount of oil recovered to an increase in the $\mathrm{pH}$ in the brine. They assert that as the salinity of the water is reduced, the divalent cations which form a bridge between the oil and the clay minerals on the surface of the reservoir rock migrate into the bulk fluid and are replaced by $\mathrm{H}^{+}$ions. This weakens the bond between the oil and the clay, resulting in an increase in the amount of oil recovered. Lager et al. (2008) propose a similar mechanism, multicomponent ionic exchange, in which the desorbed divalent ions are replaced by $\mathrm{Na}^{+}$ions in the surrounding fluid, producing a similar result. In both these mechanisms, the thickness of the thin water film separating the oil from the clay surface increases as the salinity decreases due to a decrease in the attraction between the surfaces. This results in a small amount of slip along the effective oil-clay interface, which leads to an increase in the mobility of the oil phase (Wilmott et al., 2018).

Numerous experimental studies have been conducted in order to study the various mechanisms (Austad et al., 2010; Berg et al., 2010; Lee et al., 2010; Ligthelm et al., 2009; Morrow, 1990). However, due to the complexity of the waterflooding process and the uncertainty which such complexity inevitably introduces, there is a limited understanding of how the parameters within the models for the molecular scale mechanisms affect the recovery process on the macroscopic scale. In order to study these mechanisms on the larger length scales of interest, 'upscaled' models for the various low salinity mechanisms are required.

In this paper, we use a network modelling approach to derive an upscaled model for liquid transport through a porous medium incorporating multicomponent ionic exchange. We note that, due to the similarities between this mechanism and pH increase, our approach may be easily extended to account for variations in the $\mathrm{pH}$ by modifying the expressions for the surface charge densities on the oil and solid surfaces appropriately (as we have done in Wilmott et al., 2019). In contrast to existing network models for two-phase flow (Dahle and Celia, 1999; Joekar-Niasar et al., 2010; Knudsen et al., 2002; Koplik and Lasseter, 1984; Sheng and Thompson, 2016; Thompson, 2002), we will include the effect of slip in our network model in order to understand how the salinity of the brine affects the amount of oil recovered.

There are numerous modelling approaches used to study two-phase flow through a network, which can typically be separated into three classes; quasi-static, semi-dynamic, and dynamic models. Dynamic models can be further split into the classes of 'Washburn equation' and 'annular flow' models. In $§ 1.1$, we will briefly discuss dynamic network modelling and review the appropriate literature. For a comprehensive review of alternative network modelling approaches, such as quasistatic and semi-dynamic networks, the reader is referred to Joekar-Niasar and Hassanizadeh (2012).

In $\S 2$, we will present our network model for the two-phase flow of oil and brine through a porous medium. We will assume that the salinity of the brine affects the thickness of the thin, nano-scale wetting film which separates the oil from the solid surface. We will suppose that the resulting slip along the effective oil-throat interface depends on the salinity according to the model 
derived in Wilmott et al. (2018). We will include the advection of ions in our model in order to study the effect of the salinity on the flow. We will assume that the porous medium is made up from a connected series of pores and throats, and that both phases may be present throughout the domain, with the wetting phase coating the walls of the pores and the two phases forming slugs along the lengths of the throats. Rather than keep track of the numerous interfaces separating the phases within the throats, as done by Knudsen et al. (2002), we will determine the saturation of each phase and compute the effective conductivity of the throat as a function of the saturation. We will define the phase-weighted pressure throughout the network, and formulate our model in terms of this phase-weighted pressure and also the water saturation using conservation of mass for each fluid. Formulating our model in this way will enable us to solve a single set of pressure equations, reducing the computational effort required.

In $\S 3$, we will non-dimensionalise our model and present the system of equations in vector format. We will describe a numerical scheme to solve the system subject to appropriate initial and boundary conditions. In $\S 4$, we will solve our model numerically to simulate a 'waterflood'. We will separate the waterflooding process into two stages. First, we will model a 'drainage' stage, in which oil invades an initially water-filled network through the action of capillary forces. Second, we will model a 'waterflood' stage, in which water is injected into the oil-filled network, and the oil recovered is measured. We will compare the results for a high-salinity and low-salinity waterflood on a $30 \times 30$ lattice. We will also simulate a tertiary low-salinity waterflood, in which the network is initially flooded with high salinity brine before a low-salinity injection is performed. Finally, in $\S 5$, we will discuss our findings and draw conclusions.

\subsection{Dynamic network models}

In dynamic network modelling of two-phase flow, the pores and throats of the network may be occupied by one or both phases at any given time. The pressure field in each phase, and therefore the fluid flow, is computed at each time step, and the volume fraction in each pore is updated according to the flux and saturation of the invading fluid. Dynamic network models allow partial filling of the pores or throats, and therefore require a process for tracking the saturation of each phase within the network. Some dynamic models attempt to track the interface between the fluids within the throats (for example, Al-Gharbi and Blunt, 2005; Dahle and Celia, 1999; Knudsen et al., 2002; Koplik and Lasseter, 1984), whereas others assume that the flow comprises a fraction of each phase throughout the network, with the wetting phase typically present in all pores (JoekarNiasar et al., 2010; Sheng and Thompson, 2016; Thompson, 2002). We will briefly review these two approaches and discuss their relative advantages and disadvantages.

The first approach to dynamic network modelling is to suppose that each phase spans the width of the pores and throats, and to track the interface separating the two phases, as shown in Figure 1a. The model is governed by Washburn's equation (Washburn, 1921) and conservation of flux, which read $^{1}$

$$
Q_{i j}=\frac{g_{i j}}{\mu^{\mathrm{eff}} L_{i j}}\left(p_{i}-p_{j}+P_{i j}^{c}\right), \quad \sum_{j} Q_{i j}=0,
$$

respectively, where $Q_{i j}$ is the flux through the throat connecting pores $i$ and $j, L_{i j}$ and $g_{i j}$ are the length and conductance of the throat connecting pores $i$ and $j$, respectively, $p_{i}$ is the pressure in pore $i$, and $P_{i j}^{c}$ is the capillary pressure across the oil-water interface in the throat connecting

\footnotetext{
${ }^{1}$ Note that this is simply a discretisation of Darcy's law.
} 


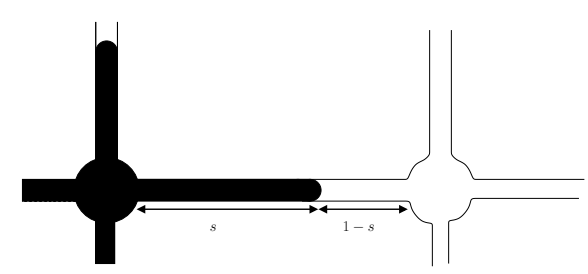

(a)

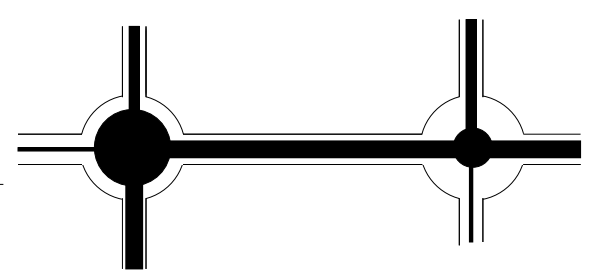

(b)

Figure 1: (a) Schematic diagram of the Washburn equation network model. (b) Schematic diagram of the annular flow network model.

pores $i$ and $j$. The capillary pressure is zero if the throat contains a single phase (since there is no interface in this case), and can be positive or negative depending on the direction of flow of the invading fluid when two phases are present. We assume that $g_{i j}$ is zero if there is no throat connecting pores $i$ and $j$, and that otherwise $g_{i j}$ depends on the geometry of the throat and is typically given by Poiseuille's law (Mortensen et al., 2005). The effective viscosity, $\mu_{i j}^{\text {eff }}$, is given by the weighted average of the viscosity of the two phases, $\mu_{i j}^{\text {eff }}=s_{i j} \mu_{o}+\left(1-s_{i j}\right) \mu_{w}$, where $s_{i j}$ is the proportion of the throat filled with the oil phase, as shown in Figure 1a.

Koplik and Lasseter (1984) use the Washburn equation to model two-phase flow in a network, and show that the problem is equivalent to current flow in an electrical resistor network. Dahle and Celia (1999) also model two-phase flow using an interface-tracking model. They restrict their model by only allowing a single interface in a throat at a time, and define a set of displacement rules at the nodes for when an interface reaches a throat entrance. They find that, in order to maintain a global mass balance, they require a correction step in their algorithm. Knudsen et al. (2002) present a two-phase dynamic network model in which the two phases form bubbles which can separate and merge together. They assume that the network is periodic, so that the volume of each fluid is conserved, in order to compute fractional flow properties of the system. Al-Gharbi and Blunt (2005) develop a dynamic interface-tracking model in which the pores and throats have triangular cross section. They track the interfaces using the effective hydraulic resistance of the throats, and include a mechanism for snap-off in their model.

The second approach to dynamic network modelling is to assume that both phases may be present throughout the network (see Joekar-Niasar et al., 2010; Sheng and Thompson, 2016; Thompson, 2002, for example). Often, this is done by assuming an 'annular flow' model, in which the non-wetting phase moves through the centre of the pores and throats, and the wetting phase is restricted to the annular region between the non-wetting phase and the pore/throat walls as shown in Figure 1b. For oil/water systems it is generally assumed that water is the wetting phase. Since both phases are present throughout the network, a separate set of equations exists for the pressure field in each phase. This is in contrast to the Washburn equation model, in which only one pressure is tracked.

The annular flow model is governed by Poiseuille's law and conservation of mass for each phase, 
and reads

$$
\begin{array}{ll}
Q_{i j}^{o}=\frac{g_{i j}^{o}}{\mu^{o}}\left(p_{j}^{o}-p_{i}^{o}\right), & V_{i} \frac{\mathrm{d} S_{i}^{o}}{\mathrm{~d} t}=-\sum_{j} Q_{i j}^{o}, \\
Q_{i j}^{w}=\frac{g_{i j}^{w}}{\mu^{w}}\left(p_{j}^{w}-p_{i}^{w}\right), & V_{i} \frac{\mathrm{d} S_{i}^{w}}{\mathrm{~d} t}=-\sum_{j} Q_{i j}^{w},
\end{array}
$$

where $Q_{i j}^{o}$ and $Q_{i j}^{w}$ are the fluxes of the oil and water, respectively, and $g_{i j}^{o}$ and $g_{i j}^{w}$ are the relative throat conductances for the oil and water phases, respectively, through the throat connecting pores $i$ and $j, p_{i}^{o}$ and $p_{i}^{j}$ are the pressures in the oil and water, respectively, $S_{i}^{o}$ and $S_{i}^{w}$ are the saturations of the oil and water, respectively, and $V_{i}$ is the volume, all in pore $i$, and $\mu^{o}$ and $\mu^{w}$ are the viscosities of the oil and water, respectively. It is worth noting that, by selecting $g_{i j}^{o, w}$ appropriately, equations (2) and (3) may be used to study fractional flow systems more generally; the annular flow model is just one physical system which this model may be used to describe.

The model $(2,3)$ can be closed by assuming the constitutive laws in which the pressure difference between the two phases in pore $i$ is given by the capillary pressure, $P_{i}^{c}$ (which is a prescribed function of the water saturation in the pore), and by assuming that the sum of the saturations equals unity, in order to ensure that the entire volume within the pore is filled with fluid. These conditions may be written as

$$
p_{i}^{o}=p_{i}^{w}+P_{i}^{c}\left(S_{i}^{w}\right), \quad S_{i}^{o}+S_{i}^{w}=1
$$

Further, the functional form for the relative throat conductance for each phase, $g_{i j}^{o}$ and $g_{i j}^{w}$, must be given. A common approach is to assume that $g_{i j}^{o}=\left(S_{i j}^{o}\right)^{2} g_{i j}$, and $g_{i j}^{w}=\left(S_{i j}^{w}\right)^{2} g_{i j} / \beta$, where $S_{i j}^{o}$ and $S_{i j}^{w}$ are the saturations of the oil and water phases in the throat connecting pores $i$ and $j$, respectively (which are usually taken to be the same as the saturations in the upstream pore), $g_{i j}$ is the conductance of the throat in the case when only a single phase is present, and the resistance factor, $\beta$, is a parameter which depends on both the boundary conditions on the fluid-solid and fluid-fluid interfaces and the geometry of the throat and is used to account for the particular physics of the problem being studied.

Thompson (2002) solves this model for a network constructed from throats with square crosssection. He solves the pressure equations separately in each phase and, by assuming that each phase is present throughout the network, reduces the need for the rule-based decision making that is often required in interface-tracking methods. Joekar-Niasar et al. (2010) compute the local capillary-pressure-saturation relationship within a cubic pore body, which they use in their model to determine the macroscopic capillary-pressure-saturation relationship. Sheng and Thompson (2016) present a unified dynamic network model which they use to study drainage and imbibition for both periodic and non-periodic networks. Their method permits the injection of both fluids simultaneously at the inlet. This enables them to study fractional flow through a non-periodic network, which they use to simulate steady-state relative permeability tests.

The main advantage of the annular flow model is that there are no interfaces to track, since each phase is present throughout the network. However, the shape parameter, $\beta$, is difficult to determine for real materials. Furthermore, this approach is of limited use to us since the annular model assumes a thick water region separating the oil from the throat surface, with thickness determined by the saturation, whereas our model for low salinity has the oil separated from the rock surface by a thin film of water, with thickness determined by the salinity. 


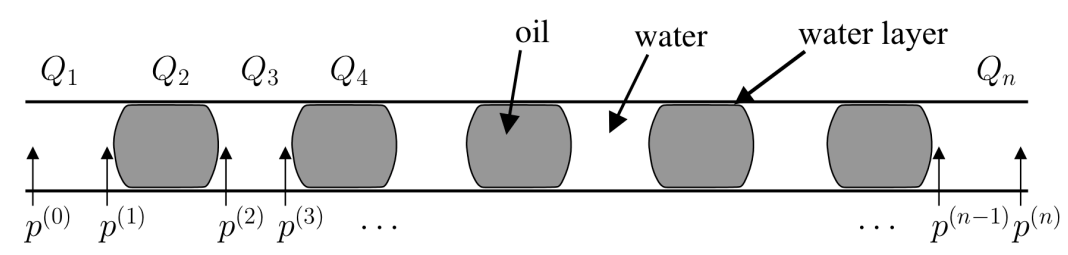

Figure 2: Schematic diagram showing the model of the pore throat, with multiple slugs along its length. The flux of each section is given by $Q_{i}$, and the pressure in the water phase at each interface is given by $p^{(i)}$.

\section{Model formulation}

In this section, we develop a network model for two-phase flow within a porous medium which combines aspects of both the Washburn equation and annular flow models. We consider a porous structure comprising throats, with uniform width, pores, that are square, and we suppose that both phases may simultaneously occupy both pores and throats. We assume that the oil phase occupies the centre of the pores, and that the water phase wets the walls of the throats and forms multiple slugs along its length, as shown in Figure 2. Between each oil slug and the wall, we assume that there is a thin film of water with thickness determined by the salinity of the adjacent water phase. Using the results in Wilmott et al. (2018), we assume that the presence of this film is seen as an increase in slip at the 'effective' oil-throat interface. We suppose that the ions are advected with the water phase.

\subsection{Throat saturation}

In order to avoid tracking multiple interfaces, we perform a local averaging procedure to formulate our model in terms the throat saturation. We consider the throat between pores $i$ and $j$ of length $L_{i j}^{t}$ and radius $R_{i j}^{t}$. We define $P^{c(l)}$ to be the capillary pressure at the $l^{\text {th }}$ interface in the throat, $p^{(l)}$ to be the pressure in the water phase at the $l^{\text {th }}$ interface in the throat, $p^{(0)}$ to be the pressure at the inlet, and $p^{(n)}$ to be the pressure at the outlet. We assume that the fluid moves due to Poiseuille's law, so that the flux of fluid $Q_{i j}^{(m)}$ in the $m^{\text {th }}$ slug is given by

$Q_{i j}^{(m)}= \begin{cases}g_{i j}^{w}\left(p^{(m-1)}-p^{(m)}\right) L_{i j}^{t} / \Delta x^{(m)}, & \text { if the } m^{\text {th }} \text { slug contains water, } \\ g_{i j}^{o}\left(\left[p^{(m-1)}+P^{c(m-1)}\right]-\left[p^{(m)}+P^{c(m)}\right]\right) L_{i j}^{t} / \Delta x^{(m)}, & \text { if the } m^{\text {th }} \text { slug contains oil }\end{cases}$

where $\Delta x^{(m)}$ is the length of the $m^{\text {th }}$ slug (so that $\sum_{m} \Delta x^{(m)}=L^{t}$ ), and

$$
g_{i j}^{w}=\frac{\pi R_{i j}^{t}{ }^{4}}{8 \mu_{w} L_{i j}^{t}}, \quad g_{i j}^{o}=\frac{\pi}{8 \mu_{o} L_{i j}^{t}}\left(R_{i j}^{t^{4}}+2 \lambda_{s i j}\left(c_{i j}^{k+}\right) R_{i j}^{t}{ }^{3}\right),
$$

are the relative conductivities of the throat in the sections containing water and oil, respectively, where $\mu_{w}$ and $\mu_{o}$ are the viscosities of the water and oil, respectively, and $\lambda_{s i j}$ is the effective slip length along the oil-throat interface which varies with the salinity, i.e. the concentration of each cation species in the throat, $c_{i j}^{k+}$, for $k=1,2$. For simplicity, we have chosen the relative conductivities (6) to be the same as for a uniform circularly cylindrical channel with and without slip (for oil and water, respectively, see Wilmott et al. (2018)). Since the focus of this paper is on the effect of salinity on the macroscopic flow, we have neglected the effect of thick wetting films 
which may occupy the corners of non-circularly cylindrical throats. This will affect the connectivity of the network, since the wetting phase may move through the corners of the pores and throats, as well as the resistivity of the two phases. To generalise the model to include this effect, as well as to study arbitrarily-shaped channels with non-uniform cross section, the functional form of (6) would need to be modified to describe the relevant physics. However, we do not consider this here.

Using the results in Wilmott et al. (2018), we choose $\lambda_{s i j}\left(c_{i j}^{k+}\right)$ to be given by the dimensionless viscosity difference, $\left(\mu_{o} / \mu_{w}-1\right)$, multiplied by the salinity-dependent thickness of the thin water film separating the oil from the clay surface, $h_{i j}^{*}\left(c_{i j}^{k+}\right)$, so that

$$
\lambda_{s i j}=\left(\frac{\mu_{o}}{\mu_{w}}-1\right) h_{i j}^{*}\left(c_{i j}^{k+}\right) .
$$

Depending on the physical model being considered, different expressions for $h^{*}$ may be appropriate. In this paper, we are interested in modelling the effect of salinity on the thickness of the water layer due to the multicomponent ionic exchange mechanism. In this mechanism, divalent cations in the water phase form a bridge between the negatively charged sites on the surfaces of the oil and the throat. As the salinity of the surrounding fluid decreases, these divalent cations migrate into the bulk fluid, and monovalent ions adsorb onto the vacated sites. This leads to a decrease in the attraction between the surfaces of the oil and the throat, which results in an increase in the thickness of the water film. Using the expression for $h_{i j}^{*}\left(c_{i j}^{k+}\right)$ in Wilmott et al. (2018), $\lambda_{s i j}$ reads

$$
\begin{aligned}
\lambda_{s i j}=\left(\frac{\mu_{o}}{\mu_{w}}-1\right) & \left(\frac{\varepsilon_{w} k_{B} T}{2\left(c_{i j}^{1+}+4 c_{i j}^{2+}\right) q^{2}}\right)^{1 / 2} \\
& \times \cosh ^{-1}\left(\frac{\sigma_{c i j} \sigma_{o i j}+\sqrt{\left(\sigma_{c i j}^{2}+2 \gamma \epsilon_{w} R_{i j}^{t} / L_{i j}^{t}{ }^{2}\right)\left(\sigma_{o}^{2}+2 \gamma \epsilon_{w} R_{i j}^{t} / L_{i j}^{t}{ }^{2}\right)}}{2 \gamma \epsilon_{w} R_{i j}^{t} / L_{i j}^{t}}\right),
\end{aligned}
$$

where $\varepsilon_{w}$, is the permittivity of the water phase, $k_{B}$ is Boltzmann's constant, $T$ is the temperature, $q$ is the absolute charge of an electron, $\gamma$ is the surface tension of the oil-water interface, and $\sigma_{c i j}$ and $\sigma_{o i j}$ are the effective surface charge densities of the capillary and oil interfaces, respectively, which are given by

$$
\sigma_{c i j}=q s_{c}^{*} \frac{K_{c}^{2} c_{i j}^{2+}-1}{1+K_{c}^{1} c_{i j}^{1+}+K_{c}^{2} c_{i j}^{2+}}, \quad \sigma_{o i j}=q s_{o}^{*} \frac{K_{o}^{2} c_{i j}^{2+}-1}{1+K_{o}^{1} c_{i j}^{1+}+K_{o}^{2} c_{i j}^{2+}},
$$

where $s_{c}^{*}$ and $s_{o}^{*}$ are the surface concentrations of cation exchange sites at the capillary and oil surfaces, respectively, $K_{c}^{1}$ and $K_{c}^{2}$ are the equilibrium constants for the reactions involving monovalent and divalent cation exchange at the capillary surface, respectively, and $K_{o}^{1}$ and $K_{o}^{2}$ are the equilibrium constants for the reactions involving monovalent and divalent cation exchange at the oil surface, respectively.

We simplify the system (5) - (9) by assuming that the radii of curvature of the advancing and receding menisci at the front and rear of the oil slugs are identical to leading $\operatorname{order}^{2}$, so that

\footnotetext{
${ }^{2}$ We are able to do this since we have assumed that the throat has uniform width.
} 
$P^{c(m-1)}=P^{c(m)}$ for all $m$, and therefore $(5)$ reads

$$
Q_{i j}^{(m)}=\left\{\begin{array}{l}
g_{i j}^{w}\left(p^{(m-1)}-p^{(m)}\right) L_{i j}^{t} / \Delta x^{(m)}, \\
g_{i j}^{o}\left(p^{(m-1)}-p^{(m)}\right) L_{i j}^{t} / \Delta x^{(m)},
\end{array}\right.
$$

which we rearrange to find that

$$
p^{(m-1)}-p^{(m)}=\left\{\begin{array}{l}
Q_{i j}^{(m)} \Delta x^{(m)} / g_{i j}^{w} L_{i j}^{t}, \quad \text { if the } m^{\text {th }} \text { slug contains water } \\
Q_{i j}^{(m)} \Delta x^{(m)} / g_{i j}^{o} L_{i j}^{t}, \quad \text { if the } m^{\text {th }} \text { slug contains oil. }
\end{array}\right.
$$

We assume that the flux is conserved along the length of the throat so that $Q_{i j}^{(m)}=Q_{i j}$, for all $m$. By summing (11) over $m$, we find that

$$
p^{(0)}-p^{(n)}=Q_{i j}\left(\frac{1}{g_{i j}^{w} L_{i j}^{t}} \sum_{\text {water }} \Delta x^{(m)}+\frac{1}{g_{i j}^{o} L_{i j}^{t}} \sum_{\text {oil }} \Delta x^{(m)}\right),
$$

where the two summations are over the values of $m$ for which the $k^{\text {th }}$ slug contain water and oil, respectively. Since we assume that the width of the channel is uniform, and $\Delta x^{(m)}$ corresponds to the length of the $m^{\text {th }}$ slug, we observe that the two summations are precisely the volume fraction of water and oil in the throat, respectively. We define the proportion of the throat filled with water, i.e. the water saturation in the throat, to be

$$
S_{i j}^{t}=\frac{1}{L_{i j}^{t}} \sum_{\text {water }} \Delta x^{(m)}
$$

Using the fact that $\sum_{\text {oil }} \Delta x^{(m)} / L_{i j}^{t}=1-S_{i j}^{t}$, we rearrange (12) to obtain an expression for the flux through the throat as a function of the water saturation and the pressure difference,

$$
Q_{i j}=\left(p^{(0)}-p^{(n)}\right) \frac{g_{i j}^{w} g_{i j}^{o}}{g_{i j}^{o} S_{i j}^{t}+g_{i j}^{w}\left(1-S_{i j}^{t}\right)} .
$$

We note that, if the capillary pressures at the advancing and receding fronts are different, each oil slug contributes an additional pressure difference. In this case, we would need to keep track of the number of oil slugs within the throat, and develop a method for determining when oil slugs are produced or destroyed. This is important because it may contribute to the low salinity effect (Drummond and Israelachvili, 2004); however we do not address this here.

Instead of keeping track of the number of oil slugs within the throat, we keep track of the saturation and assume that the flux of each phase is given by the saturation of the phase multiplied by the flux through the throat. Thus, in our network model, we write the flux of each phase through the throat connecting pores $i$ and $j$ as

$$
Q_{i j}^{w}=S_{i j}^{t} Q_{i j}, \quad Q_{i j}^{o}=\left(1-S_{i j}^{t}\right) Q_{i j}
$$

where the $w$ and $o$ superscripts correspond to the water phase and oil phase, respectively, and the total flux $Q_{i j}$ is related to the throat saturation, $S_{i j}^{t}$, using (14). 


\subsection{Phase-weighted pressure}

Since we assume that there is a pressure difference between the two phases in the pores resulting from the capillary pressure, $p^{(0)}$ and $p^{(n)}$ depend on which phase is entering and leaving the throat at any given time. Since we track the saturation (rather than the position of each phase) within the throat, we take the pressures at the inlet and outlet to be the weighted averages of the pressures in each phase. We choose the weighting to be given by the saturation in the neighbouring pore so that, for the throat connecting pores $i$ and $j$, the inlet and outlet pressures are given by

$$
p^{(0)}=S_{i}^{w} p_{i}^{w}+S_{i}^{o} p_{i}^{o}, \quad p^{(n)}=S_{j}^{w} p_{j}^{w}+S_{j}^{o} p_{j}^{o},
$$

respectively, where $S_{k}^{w}$ and $S_{k}^{o}$ are the saturations of water and oil, respectively, and $p_{k}^{w}$ and $p_{k}^{o}$ are the pressures in the water and oil phases, respectively, in pore $k$. By substituting $p_{k}^{o}=p_{k}^{w}+P_{k}^{c}$ and $S^{o}+S^{w}=1$ into (16), and defining $S_{k}:=S_{k}^{w}$, we find that

$$
p^{(0)}=p_{i}^{w}+\left(1-S_{i}\right) P_{i}^{c}\left(S_{i}\right), \quad p^{(n)}=p_{j}^{w}+\left(1-S_{j}\right) P_{j}^{c}\left(S_{j}\right),
$$

where the capillary-pressure-saturation relationship, $P_{k}^{c}\left(S_{k}\right)$, depends on the geometry of the pore.

\subsection{Governing equations}

We simplify the notation by defining

$$
P_{k}=p_{k}^{w}+\left(1-S_{k}\right) P_{k}^{c}\left(S_{k}\right),
$$

to be the phase-weighted pressure at node $k$. Substituting (17) into (14), and using (18), the total flux through the throat connecting pores $i$ and $j$ becomes

$$
Q_{i j}=\left(P_{i}-P_{j}\right) \frac{g_{i j}^{w} g_{i j}^{o}}{g_{i j}^{o} S_{i j}^{t}+g_{i j}^{w}\left(1-S_{i j}^{t}\right)} .
$$

We assume that the net flux through each pore is conserved, and that the rate of change of the volume of water in a pore is given by the net flux of water into the pore. At pore $i$, our model reads

$$
\sum_{j} Q_{i j}=0, \quad V_{i} \frac{\mathrm{d} S_{i}}{\mathrm{~d} t}=-\sum_{j} S_{i j}^{t} Q_{i j},
$$

where $V_{i}$ is the volume of the $i^{\text {th }}$ pore, and we perform the summation over the values of $j$ for which pores $i$ and $j$ are connected by a throat. We only need to solve for the saturation of the water phase, since the saturation of the oil phase in pore $i$ is given by $1-S_{i}$.

In order for oil to enter the throat connecting pores $i$ and $j$, the capillary pressure in the upstream pore must exceed an entry pressure, $p_{i j}^{e}$, which is determined by the geometry of the throat and the surface tension of the oil-water interface. A model for how the oil phase enters the pore throats is required in order to determine how the entry pressure depends on the slip length, however this is beyond the scope of this paper. For simplicity, we assume that the entry pressure decreases as the slip length increases according to

$$
p_{i j}^{e}=\frac{2 \gamma}{R_{i j}^{t}+2 \lambda_{s i j}\left(c_{i j}^{k+}\right)} .
$$


We assume that the saturation in the throat is given by the saturation in the upstream pore if the capillary pressure exceeds the entry pressure, otherwise the throat is entirely filled with water. The upstream pore is determined by the sign of $P_{i}-P_{j}$, so that

$$
S_{i j}^{t}= \begin{cases}S_{i}, & \text { if } P_{i}>P_{j} \text { and } P_{i}^{c}\left(S_{i}\right)>p_{i j}^{e}\left(c_{i j}^{k+}\right), \\ S_{j}, & \text { if } P_{i}<P_{j} \text { and } P_{j}^{c}\left(S_{j}\right)>p_{i j}^{e}\left(c_{i j}^{k+}\right) \\ 1, & \text { otherwise }\end{cases}
$$

We assume that the rate of change of the total number of each ion species in a pore is equal to the net flux of ions into the pore, and that the concentration of ions in the throat is given by the concentration in the upstream pore. This reads

$$
V_{i} \frac{\mathrm{d}\left(c_{i}^{k+} S_{i}\right)}{\mathrm{d} t}=-\sum_{j} c_{i j}^{k+} S_{i j}^{t} Q_{i j}, \quad c_{i j}^{k+}= \begin{cases}c_{i}^{k+}, & \text { if } P_{i}>P_{j}, \\ c_{j}^{k+}, & \text { if } P_{i}<P_{j},\end{cases}
$$

where $c_{i}^{k+}$ is the concentration of each ion species in pore $i$, for $k=1,2$, and we perform the summation over the values of $j$ for which pores $i$ and $j$ are connected by a throat.

Finally, we assume that the capillary-pressure-saturation function, $P_{i}^{c}$, depends on the geometry of the pore. If we assume that the pores are square, we find (see Appendix A) that

$$
P_{i}^{c}=\frac{2 \gamma}{R_{i}^{p}} \sqrt{\frac{4-\pi}{S_{i}}}
$$

where $2 R_{i}^{p}$ is the length of each side of the pore.

\subsection{Boundary conditions}

The initial and boundary conditions depend on the particular problem being studied. In $\S 4$, we will study drainage and waterflooding for a simple two-dimensional grid network, and discuss the appropriate initial and boundary conditions for each situation. However, in both cases, we will assume that the outlet nodes are subject to a prescribed reference pressure, $\tilde{P}$. We prescribe the pressure and saturation at the inlet nodes to be $S_{i}^{\text {in }}$ and $\tilde{P}+P_{i}^{\text {in }}$ respectively, which may be different at each node in order to ensure that the pressure in each phase is consistent across the inlet. We also assume that the concentration of ions at the inlet are prescribed functions of time, $c_{i}^{k+}=c_{\mathrm{in}}^{k+}(t)$, to allow us to study, for example, a high-salinity waterflood followed by a low-salinity waterflood. The boundary conditions thus read

$$
\begin{array}{ll}
S_{i}=S_{i}^{\text {in }}, \quad P_{i}=\tilde{P}+P_{i}^{\text {in }}, \quad c_{i}^{k+}=c_{\mathrm{in}}^{k+}(t), & \text { for nodes } i \text { connected to the inlet, } \\
P_{i}=\tilde{P}, & \text { for nodes } i \text { connected to the outlet. }
\end{array}
$$

We prescribe an initial saturation at each node, $S_{I i}$, and an initial ion concentration throughout the network, $c_{I}^{k+}$, so that

$$
S_{i}=S_{I i}, \quad c_{i}^{k+}=c_{I}^{k+}, \quad \text { at } t=0 .
$$

Equations (19) - (27) defines a system of equations for $c_{i}^{k+}, S_{i}$, and $P_{i}$, from which we can compute $p_{i}^{w}$ and $p_{i}^{o}$. 


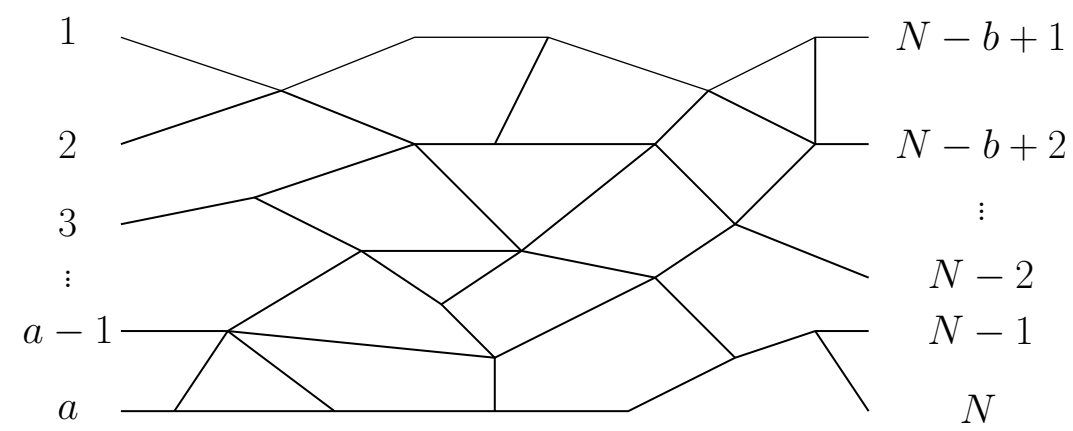

Figure 3: Schematic diagram of the network comprising $N$ nodes, with $a$ inlet nodes and $b$ outlet nodes.

\section{Model solution}

We wish to solve the two-phase flow network model presented in $\S 2$ for a network consisting of $N$ pores, including $a$ inlets, and $b$ outlets, as shown in Figure 3. We suppose that the pores (represented by nodes in our network) are square, with pore $i$ having side length $2 R_{i}^{p}$, and we suppose that the radius and length of the throat connecting pores $i$ and $j$ (represented by an edge in our network) are given by $R_{i j}^{t}$ and $L_{i j}^{t}$, respectively. We define $R^{p}, R^{t}$, and $L^{t}$, to be typical values for $R_{i}^{p}, R_{i j}^{t}$, and $L_{i j}^{t}$, respectively.

The two-phase flow through the internal nodes, $a<i \leq N-b$, of the network is governed by equations (19) - (24), with the conductivities of the throats given by (6). Substituting (19) into (20) and (23), the system reads

$$
\begin{aligned}
\sum_{i} G_{i j}\left(P_{i}-P_{j}\right) & =0, & & \text { for } a<i \leq N-b, \\
V_{i} \frac{\mathrm{d} S_{i}}{\mathrm{~d} t} & =-\sum_{j} S_{i j}^{t} G_{i j}\left(P_{i}-P_{j}\right), & & \text { for } a<i \leq N-b, \\
V_{i} \frac{\mathrm{d}\left(c_{i}^{k+} S_{i}\right)}{\mathrm{d} t} & =-\sum_{j} c_{i j}^{k+} S_{i j}^{t} G_{i j}\left(P_{i}-P_{j}\right), & & \text { for } a<i \leq N-b,
\end{aligned}
$$

subject to

$$
\begin{aligned}
& S_{i}=S_{i}^{\text {in }}, \quad P_{i}=\tilde{P}+P_{i}^{\text {in }}, \quad c_{i}^{k+}=c_{\text {in }}^{k+}(t), \quad \text { for } 1 \leq i \leq a, \\
& P_{i}=\tilde{P}, \quad \text { for } N-b<i \leq N \text {, } \\
& S_{i}=S_{I i}, \quad c_{i}^{k+}=c_{I}^{k+}, \quad \text { at } t=0,
\end{aligned}
$$

where

$$
G_{i j}=\frac{\pi R_{i j}^{t}{ }^{4}}{8 L_{i j}^{t}} \frac{1+2 \frac{\lambda_{s i j}}{R_{i j}^{t}}}{\mu_{w}\left(1+2 \frac{\lambda_{s i j}}{R_{i j}^{t}}\right) S_{i j}^{t}+\mu_{o}\left(1-S_{i j}^{t}\right)},
$$

and $\lambda_{s i j}, p_{i j}^{e}, S_{i j}^{t}, c_{i j}^{k+}$, and $P_{i}^{c}$ are given by (8) and (21) - (24). 


\subsection{Non-dimensionalisation}

We define $\mathcal{P}$ to be a typical size for the inlet pressures, $P_{i}^{\text {in }}$, which we will define explicitly in $\S 4.1$ and $\S 4.2$ when we come to study drainage and waterflooding models, respectively. We use hats to denote dimensionless variables, and non-dimensionalise the model using the scalings

$$
\begin{gathered}
P_{i}=\tilde{P}+\mathcal{P} \hat{P}_{i}, \quad P_{i}^{\mathrm{in}}=\tilde{P}+\mathcal{P} \hat{P}_{i}^{\mathrm{in}}, \quad P_{i}^{c}=\mathcal{P} \hat{P}_{i}^{c} \quad p_{i j}^{e}=\mathcal{P} \hat{p}_{i j}^{e}, \\
R_{i}^{p}=R^{p} \hat{R}_{i}^{p}, \quad R_{i j}^{t}=R^{t} \hat{R}_{i j}^{t}, \quad \lambda_{s i j}=R^{t} \hat{\lambda}_{s i j}, \quad L_{i j}^{t}=L^{t} \hat{L}_{i j}^{t}, \quad t=\frac{8 \mu_{w} L^{t}\left(R^{p}\right)^{3}}{\pi\left(R^{t}\right)^{4} \mathcal{P}} \hat{t} \\
V_{i}=\left(R^{p}\right)^{3} \hat{V}_{i}, \quad G_{i j}=\frac{\pi\left(R^{t}\right)^{4}}{8 \mu_{w} L^{t}} \hat{G}_{i j}, \quad \sigma_{c i j}=q s_{c}^{*} \hat{\sigma}_{c i j} \quad \sigma_{o i j}=q s_{o}^{*} \hat{\sigma}_{o i j} \\
c_{i}^{k+}=c_{I}^{k+} \hat{c}_{i}^{k+}, \quad c_{i j}^{k+}=c_{I}^{k+} \hat{c}_{i j}^{k+}, \quad c_{\mathrm{in}}^{k+}=c_{I}^{k+} \hat{c}_{\mathrm{in}}^{k+} .
\end{gathered}
$$

Writing (28) - (34) in terms of the dimensionless variables, and dropping the hat notation, yields

$$
\begin{aligned}
\sum_{i} G_{i j}\left(P_{i}-P_{j}\right) & =0, & & \text { for } a<i \leq N-b, \\
V_{i} \frac{\mathrm{d} S_{i}}{\mathrm{~d} t} & =-\sum_{j} S_{i j}^{t} G_{i j}\left(P_{i}-P_{j}\right), & & \text { for } a<i \leq N-b, \\
V_{i} \frac{\mathrm{d}\left(c_{i}^{k+} S_{i}\right)}{\mathrm{d} t} & =-\sum_{j} c_{i j}^{k+} S_{i j}^{t} G_{i j}\left(P_{i}-P_{j}\right), & & \text { for } a<i \leq N-b,
\end{aligned}
$$

subject to

$$
\begin{aligned}
& S_{i}=S_{i}^{\mathrm{in}}, \quad P_{i}=P_{i}^{\mathrm{in}}, \quad c_{i}^{k+}=c_{\mathrm{in}}^{k+}(t), \quad \text { for } 1 \leq i \leq a, \\
& P_{i}=0 \text {, } \\
& S_{i}=S_{I i}, \quad c_{i}^{k+}=1, \\
& \text { for } N-b<i \leq N \text {, } \\
& \text { at } t=0 \text {, }
\end{aligned}
$$


where

$$
\begin{aligned}
& G_{i j}=\frac{R_{i j}^{t}{ }^{4}}{L_{i j}^{t}} \frac{1+2 \frac{\lambda_{s i j}}{R_{i j}^{t}}}{\left(1+2 \frac{\lambda_{s i j}}{R_{i j}^{t}}\right) S_{i j}^{t}+\mu^{*}\left(1-S_{i j}^{t}\right)}, \\
& S_{i j}^{t}= \begin{cases}S_{i}, & \text { if } P_{i}>P_{j} \text { and } P_{i}^{c}\left(S_{i}\right)>p_{i j}^{e}, \\
S_{j}, & \text { if } P_{i}<P_{j} \text { and } P_{j}^{c}\left(S_{j}\right)>p_{i j}^{e} \\
1, & \text { otherwise }\end{cases} \\
& p_{i j}^{e}=\frac{2 \Gamma_{\mathcal{P}}}{R_{i j}^{t}+2 \lambda_{s i j}}, \\
& c_{i j}^{k+}= \begin{cases}c_{i}^{k+}, & \text { if } P_{i}>P_{j}, \\
c_{j}^{k+}, & \text { if } P_{i}<P_{j},\end{cases} \\
& \lambda_{s i j}=\frac{\epsilon_{2}\left(\mu^{*}-1\right)}{\left(c_{i j}^{1+}+4 c^{*} c_{i j}^{2+}\right)^{1 / 2}} \cosh ^{-1}\left(\frac{s^{*} \sigma_{c i j} \sigma_{o i j}+\sqrt{\left(\sigma_{c i j}^{2}+\Gamma R_{i j}^{t} / L_{i j}^{t}{ }^{2}\right)\left(s^{* 2} \sigma_{o i j}^{2}+\Gamma R_{i j}^{t} / L_{i j}^{t}{ }^{2}\right)}}{\Gamma R_{i j}^{t} / L_{i j}^{t}{ }^{2}}\right), \\
& P_{i}^{c}=\frac{2 \Gamma_{\mathcal{P}} R^{*}}{R_{i}^{p}} \sqrt{\frac{4-\pi}{S_{i}} .}
\end{aligned}
$$

We have introduced seven nondimensional parameters, $\mu^{*}, s^{*}, \epsilon_{2}, c^{*}, R^{*}, \Gamma$ and $\Gamma_{\mathcal{P}} ; \mu^{*}=\mu_{o} / \mu_{w}$ is the viscosity ratio of the oil to the water, $s^{*}=s_{o}^{*} / s_{c}^{*}$ is the ratio of the surface concentration of cation exchange sites on the oil surface to the surface concentration of cation exchange sites on the surface of the capillary, $\epsilon_{2}=\left(\varepsilon_{w} k_{B} T / 2 c_{I}^{1+} q^{2}\right)^{1 / 2} / R^{t}$ is the dimensionless Debye length, $c^{*}=c_{I}^{2+} / c_{I}^{1+}$ is the ratio of divalent ions to monovalent ions, $R^{*}=R^{t} / R^{p}$ is the ratio of a typical throat radius to a typical pore radius, $\Gamma=2 \gamma \varepsilon_{w} R^{t} / L^{t^{2}} q^{2} s_{c}^{* 2}$ is the ratio of capillary forces to electrostatic forces, and $\Gamma_{\mathcal{P}}=\gamma / R^{t} \mathcal{P}$ is the ratio of the capillary pressure to the applied pressure.

Hadia et al. (2012) performed core flood experiments with monovalent and divalent cation concentrations given by $c_{\text {low }}^{1+} \approx 15 \mathrm{~mol} \mathrm{~m}^{-3}$ and $c_{\text {low }}^{2+} \approx 0 \mathrm{~mol} \mathrm{~m}^{-3}$, respectively, in the low salinity regime, and $c_{\text {high }}^{1+} \approx 600 \mathrm{~mol} \mathrm{~m}^{-3}$ and $c_{\text {high }}^{2+} \approx 140 \mathrm{~mol} \mathrm{~m}^{-3}$, respectively, in the high salinity regime. We assume that, initially, the concentration of ions is given by the high-salinity regime, so that $c_{I}^{k+}=c_{h i g h}^{k+}$. A full list of the dimensional and dimensionless parameter values are given in Table 1 and Table 2 , respectively. We will select $\mathcal{P}$, and therefore determine $\Gamma_{\mathcal{P}}$, differently depending on whether we are studying a drainage or waterflood problem, and will discuss each case in $\S 4$.

\subsection{Vector formulation}

We write $\mathbf{P}, \mathbf{S}$, and $\mathbf{c}^{k+}$ as the column vector forms of $P_{i}, S_{i}$, and $c_{i}^{k+}$, respectively, which we separate into the components

$$
\mathbf{P}=\left(\mathbf{P}_{L} ; \mathbf{P}_{M} ; \mathbf{P}_{R}\right), \quad \mathbf{S}=\left(\mathbf{S}_{L} ; \mathbf{S}_{M} ; \mathbf{S}_{R}\right), \quad \mathbf{c}^{k+}=\left(\mathbf{c}^{k+}{ }_{L} ; \mathbf{c}^{k+}{ }_{M} ; \mathbf{c}_{R}^{k+}\right),
$$

where we have used the subscripts $L, M$, and $R$ to denote left, middle, and right, respectively, and the corresponding vectors have length $a, N-a-b$, and $b$, respectively. 


Paramete
$R^{p}$
$R^{t}$
$L^{t}$
$\gamma$
$\mu_{o}$
$\mu_{w}$
$c_{l o w}^{1+}$
$c_{h i g h}^{1+}$
$c_{\text {low }}^{2+}$
$c_{h i g h}^{2+}$
$s_{c}^{*}$
$s_{o}^{*}$
$K_{c}^{1}$
$K_{c}^{2}$
$K_{o}^{1}$
$K_{o}^{2}$
$\varepsilon_{w}^{2}$
$T$
$q$
$k_{B}$

Value
$2 \mu \mathrm{m}$
$1 \mu \mathrm{m}$
$10 \mu \mathrm{m}$
$0.02 \mathrm{~J} \mathrm{~m}^{-2}$
$0.01 \mathrm{~kg} \mathrm{~m}^{-1} \mathrm{~s}^{-1}$
$8.9 \times 10^{-4} \mathrm{~kg} \mathrm{~m}^{-1} \mathrm{~s}^{-1}$
$15 \mathrm{~mol} \mathrm{~m}^{-3}$
$600 \mathrm{~mol} \mathrm{~m}^{-3}$
$0 \mathrm{~mol} \mathrm{~m}^{-3}$
$140 \mathrm{~mol} \mathrm{~m}^{-3}$
$10^{16} \mathrm{~m}^{-2}$
$7.5 \times 10^{17} \mathrm{~m}^{-2}$
$8 \times 10^{3} \mathrm{~m}^{3} \mathrm{~mol}^{-1}$
$9.7 \times 10^{3} \mathrm{~m}^{3} \mathrm{~mol}^{-1}$
$6 \times 10^{-4} \mathrm{~m}^{3} \mathrm{~mol}^{-1}$
$3 \times 10^{-4} \mathrm{~m}^{3} \mathrm{~mol}^{-1}$
$5 \times 10^{-10} \mathrm{C}^{2} \mathrm{~J}^{-1} \mathrm{~m}^{-1}$
$375^{\circ} \mathrm{K}^{1}$
$1.6 \times 10^{-19} \mathrm{C}^{-23} \mathrm{~J} \mathrm{~K}^{-1}$
$1.4 \times 10^{-23}$

Reference/Notes

(Nelson, 2009)

(Nelson, 2009)

(Nelson, 2009)

(Buckley, 1996)

(Buckley, 1996)

(Buckley, 1996)

(Hadia et al., 2012)

(Hadia et al., 2012)

(Hadia et al., 2012)

(Hadia et al., 2012)

( $\mathrm{Li}$ and $\mathrm{Xu}, 2008)$

(Lewis, 1937)

(Fletcher and Sposito, 1989)

(Fletcher and Sposito, 1989)

(Fournier et al., 1998)

(Joseph, 1946)

(Malmberg and Maryott, 1956)

(Lager et al., 2008)

Table 1: Dimensional parameters

$\begin{array}{ccc}\text { Parameter } & \text { Expression } & \text { Value } \\ \mu^{*} & \mu_{o} / \mu_{w} & 11 \\ s^{*} & s_{o}^{*} / s_{c}^{*} & 75 \\ \epsilon_{2} & \left(\varepsilon_{w} k_{B} T / 2 c_{I}^{1+} q^{2}\right)^{1 / 2} / R^{t} & 2.5 \times 10^{-3} \\ c^{*} & c_{I}^{2+} / c_{I}^{1+} & 0.3 \\ R^{*} & R^{t} / R^{p} & 0.5 \\ \Gamma & 2 \gamma \varepsilon_{w} R^{t} / L^{t^{2}} q^{2} s_{c}^{* 2} & 0.08 \\ \Gamma_{\mathcal{P}} & \gamma / R^{t} \mathcal{P} & \end{array}$

Table 2: Dimensionless parameters 
Denoting $\mathbf{V}=\left(\mathbf{V}_{L}, \mathbf{V}_{M}, \mathbf{V}_{R}\right)$ as the vector form of $V_{i}$, we write (36) - (38) in the form

$$
\begin{gathered}
\boldsymbol{L} \mathbf{P}=\mathbf{0}, \\
\mathbf{V}_{M} \circ \frac{\mathrm{d} \mathbf{S}_{M}}{\mathrm{~d} t}=-\boldsymbol{L}_{S} \mathbf{P}, \quad \mathbf{V}_{M} \circ \frac{\mathrm{d}\left(\mathbf{c}_{M}^{k+} \circ \mathbf{S}_{M}\right)}{\mathrm{d} t}=-\boldsymbol{L}_{c}^{k+} \mathbf{P},
\end{gathered}
$$

where $\circ$ denotes element-wise multiplication, and

$$
\begin{aligned}
(\boldsymbol{L})_{i-a, j} & =\delta_{i-a, j}\left(\sum_{k} G_{i-a, k}\right)-G_{i-a, j} & & \text { for } a<i \leq N-b, \\
\left(\boldsymbol{L}_{S}\right)_{i-a, j} & =\delta_{i-a, j}\left(\sum_{k} S_{i-a, k}^{t} G_{i-a, k}\right)-S_{i-a, j}^{t} G_{i-a, j} & & \text { for } a<i \leq N-b, \\
\left(\boldsymbol{L}_{c}^{k+}\right)_{i-a, j} & =\delta_{i-a, j}\left(\sum_{l} c_{i-a, l}^{k+} S_{i-a, l}^{t} G_{i-a, l}\right)-c_{i-a, j}^{k+} S_{i-a, j}^{t} G_{i-a, j} & & \text { for } a<i \leq N-b,
\end{aligned}
$$

correspond to the internal rows of the weighted Laplacian matrices for the conductivity of the throats, the water saturation multiplied by the conductivity of the throats (i.e. the conductivity of the water phase), and the concentrations of the different ion species multiplied by the conductivity of the water phase (i.e. the ion conductivites), respectively.

We separate the non-square $(N-a-b) \times N$ matrix, $\boldsymbol{L}$, into the following components,

$$
\left.\boldsymbol{L}=(\underbrace{\boldsymbol{L}_{1}}_{a}|\underbrace{\boldsymbol{L}_{2}}_{N-a-b}| \underbrace{\boldsymbol{L}_{3}}_{b})\right\}_{N-a-b}
$$

where the dimensions of $\boldsymbol{L}_{1}, \boldsymbol{L}_{2}$ and $\boldsymbol{L}_{3}$ have been shown. We substitute (54) into (49) and rearrange to obtain

$$
\mathbf{P}_{M}=-\boldsymbol{L}_{2}^{-1} \boldsymbol{L}_{1} \mathbf{P}_{L}
$$

which, along with (50), defines a system of equations for $\mathbf{P}_{M}, \mathbf{S}_{M}$, and $\mathbf{c}_{M}^{k+}$. We solve (50) and (55) subject to the boundary and initial conditions (39) - (41), which read

$$
\begin{aligned}
& \mathbf{P}_{L}=\mathbf{P}^{\text {in }}, \quad \mathbf{P}_{R}=\mathbf{0}, \quad \mathbf{S}_{L}=\mathbf{S}^{\text {in }}, \quad \mathbf{c}_{L}^{k+}=c_{i n}^{k+} \cdot \mathbf{1}, \\
& \mathbf{S}_{M}=\mathbf{S}_{I M}, \quad \mathbf{c}^{k+}=1, \quad \text { at } t=0,
\end{aligned}
$$

where we have written $\mathbf{P}^{\text {in }}, \mathbf{S}^{\text {in }}$, and $\mathbf{S}_{I}=\left(\mathbf{S}_{I L} ; \mathbf{S}_{I M} ; \mathbf{S}_{I R}\right)$ as the vector forms of $P_{i}^{\text {in }}, S_{i}^{\text {in }}$, and $S_{I i}$, respectively.

Since $\boldsymbol{L}_{1}, \boldsymbol{L}_{2}, \boldsymbol{L}_{S}$, and $\boldsymbol{L}_{c}^{k+}$ depend non-linearly on $\mathbf{P}, \mathbf{S}$, and $\mathbf{c}^{k+}$ through equations (42) (47), we solve (50), (55), and (57) using an implicit scheme for the pressure, and an explicit scheme for the saturation and ion concentrations along with an adaptive time step. At each time step, we compute $\boldsymbol{L}_{1}, \boldsymbol{L}_{2}, \boldsymbol{L}_{S}$, and $\boldsymbol{L}_{c}^{k+}$ using the solutions for $\mathbf{P}, \mathbf{S}$, and $\mathbf{c}^{k+}$ from the previous time step, and then we determine $\mathbf{P}_{M}$ from (55) and use this updated solution for $\mathbf{P}_{M}$ in order to compute the saturations and concentrations using (50). Denoting the $n^{\text {th }}$ time step using a superscript, and letting $\Delta t^{n}$ be the size of the $n^{\text {th }}$ time step, this process reads

$$
\begin{gathered}
\mathbf{P}_{M}^{n+1}=-\left(\boldsymbol{L}_{2}^{n}\right)^{-1}\left(\boldsymbol{L}_{1}^{n} \mathbf{P}_{L}\right), \quad \mathbf{S}_{M}^{n+1}=\mathbf{S}_{M}^{n}-\Delta t^{n}\left(\mathbf{L}_{S}^{n} \mathbf{P}^{n+1}\right) \oslash \mathbf{V}_{M}, \\
\mathbf{c}_{M}^{k+}{ }^{n+1} \mathbf{S}_{M}^{n+1}=\mathbf{c}_{M}^{k+}{ }^{n} \mathbf{S}_{M}^{n}-\Delta t^{n}\left(\boldsymbol{L}_{c}^{k+}{ }^{n} \mathbf{P}^{n+1}\right) \oslash V_{M},
\end{gathered}
$$


with the initial conditions

$$
\mathbf{S}_{M}^{0}=\mathbf{S}_{I M}, \quad \mathbf{c}_{M}^{k+}{ }^{0}=\mathbf{1},
$$

where $\oslash$ denotes element-wise division. We choose the time step, $\Delta t^{n}$, according to

$$
\Delta t^{n}=\frac{1}{h} \min \left(\Delta t_{0}^{n}, \Delta t_{1}^{n}\right)
$$

where

$$
\Delta t_{0}^{n}=\min \left(\left[\mathbf{V}_{M} \circ \mathbf{S}_{M}^{n} \oslash\left(\boldsymbol{L}_{S}^{n} \mathbf{P}^{n+1}\right)\right]_{i}\right), \quad \text { for } i \text { s.t. }\left(\boldsymbol{L}_{S}^{n} \mathbf{P}^{n+1}\right)_{i}>0,
$$

and

$$
\Delta t_{1}^{n}=\min \left(\left[\mathbf{V}_{M} \circ\left(\mathbf{S}_{M}^{n}-1\right) \oslash\left(\boldsymbol{L}_{S}^{n} \mathbf{P}^{n+1}\right)\right]_{i}\right), \quad \text { for } i \text { s.t. }\left(\boldsymbol{L}_{S}^{n} \mathbf{P}^{n+1}\right)_{i}<0,
$$

are the smallest time steps over which one of the pores within the network jumps from its current state to being saturated with oil or water, $S=0$ and $S=1$, respectively. Defining $\Delta t^{n}$ in this way ensures that the saturation in each node stays strictly between 0 and 1 , preventing numerical issues which may occur if an unphysical saturation develops. We choose $h=10$ in our numerical simulations. It is worth noting that, while this approach works for small networks, larger systems of practical interest require a more sophisticated numerical scheme since it is unlikely to be viable to relate the numerical timestep to the time for the next invasion step in this case. Alternatively, network homogenisation techniques (see, for example, Chapman and Shabala, 2017) may be utilised in order to reduce the network model to a system of ODEs; however this is beyond the scope of this paper.

By formulating our problem in terms of an implicit pressure explicit saturation scheme, we have removed the non-linearities from the pressure equation (58). However, since the solution at each time step now depends on the solution from the previous time step, we find that we no longer have a well-defined initial condition. We need to know the pressure field in order to determine the direction of flow and, consequently, to determine $S_{i j}^{t}$ and $c_{i j}^{k+}$ at $t=0$ using (43) and (45). We can get around this issue by supposing that the throat saturations and ion concentrations are initially equal to the mean values of the neighbouring pores, $S_{i j}^{t}=\frac{1}{2}\left(S_{i}+S_{j}\right)$ and $c_{i j}^{k+}=\frac{1}{2}\left(c_{i}^{k+}+c_{j}^{k+}\right)$ at $t=0$, to remove the pressure dependence from the initial condition. While this may not be physically accurate, we found during our numerical simulations that the appropriate directions of flow in the throats, and therefore the correct throat saturations and ion concentrations, stabilise within a few time steps.

In $\S 4$, we simulate a waterflood on an oil-filled network by numerically solving equations (58) (63). We define an appropriate two-stage process, in which an initially water-saturated network is flushed with oil before a waterflood injection is performed, and we define the initial and boundary conditions for each stage of this process.

\section{Waterflood simulation}

In this section, we simulate a waterflood on an oil-filled network by numerically solving (58) (60). The naive initial condition to apply in a waterflood simulation is that the water saturation, $S_{k}$, is equal to some small prescribed value everywhere. However, we find that, when we impose such a condition, it is unsteady due the differences between the capillary pressures in the differentsized pores. This can take some time to settle during a waterflood simulation, which affects the results and can produce unwanted effects such as flow reversal. To prevent this issue, we follow 
Held and Celia (2001) and Joekar-Niasar et al. (2008) and separate the waterflooding process into two stages.

We first replicate the initial formation of the oil reservoir, in which the oil phase slowly propagates through the water-filled reservoir rock. We connect oil to the inlet nodes of the network and prescribe a high pressure in the oil phase so that the oil invades the pores. We numerically solve our model until a small volume of water remains and the saturation in each pore reaches a steady state. We refer to this as the 'drainage' stage.

We then use the steady-state solution found from the drainage stage as the initial condition for the 'waterflood' stage (sometimes referred to as imbibition), in which we inject water in one end of the network until the saturation in each pore of the network reaches a steady state. We study two cases of waterflooding. First, we perform a secondary-stage waterflood, in which either high salinity or low salinity water is injected, and we compare the amount of oil recovered in each case. Second, we study the case in which the network is initially flooded with high-salinity water, before a low-salinity water is injected. This is known as a tertiary recovery process, in which the low salinity brine is used to obtain additional oil after the high-salinity waterflood has been performed.

\subsection{Drainage stage}

Since drainage occurs due to capillary forces alone, we assume that the pressure in the water phase, $p_{i}^{w}$, may be set to be zero without loss of generality at the inlets and outlet nodes of the network. We suppose that the network is initially water-filled, and that the inlet nodes are connected to a bath of oil held at constant 'drainage' pressure ${ }^{3}, P_{D}$, so that the capillary pressure in the inlet nodes is $P_{i}^{c}=P_{D}$. Therefore, the saturation in the inlet nodes is found by inverting the capillary pressure function, $S_{i}^{\text {in }}=\left(P_{i}^{c}\right)^{-1}\left(P_{D}\right)$, for $1 \leq i \leq a$. For simplicity, we assume that the ion concentrations are constant (given by the high salinity regime) throughout the network during the drainage stage, and therefore set $\lambda_{s}=0$ and neglect (59). Recalling from (18) that $P_{i}=p_{i}^{w}+\left(1-S_{i}\right) P_{i}^{c}\left(S_{i}\right)$, we find that the dimensional inlet pressures are given by

$$
P_{i}^{\text {in }}=\left[1-\left(P_{i}^{c}\right)^{-1}\left(P_{D}\right)\right] P_{D}
$$

We set our pressure scale to be $\mathcal{P}=P_{D}$, so that the full set of dimensionless initial and boundary conditions read

$$
\begin{gathered}
\mathbf{S}_{I M}=\mathbf{1}, \quad \text { at } t=0, \\
P_{i}^{\text {in }}=1-\left(P_{i}^{c}\right)^{-1}(1), \quad S_{i}^{\text {in }}=\left(P_{i}^{c}\right)^{-1}(1), \quad \text { for } 1 \leq i \leq a .
\end{gathered}
$$

We generate appropriate networks on a regular $30 \times 30$ grid (so that $N=900$ and $a=b=30$ ) by selecting the pore sizes, $R_{i}^{p}$, from a uniform distribution on [0,2], and the throat sizes, $R_{i j}^{t}$, from a uniform distribution on $\left[0,\left(1 / R^{*}\right) \min \left\{R_{i}^{p}, R_{j}^{p}\right\}\right]$. This ensures that the average pore radius is unity, and that the radii of the throats are always smaller than the radii of the adjacent pores. By balancing (47) with (66) for a water saturation of $O(0.1)$, we choose our pressure scale to be $\mathcal{P}=P_{D}=10^{5} \mathrm{~kg} \mathrm{~m}^{-1} \mathrm{~s}^{-2}$, and find that $\Gamma_{\mathcal{P}} \approx 0.2$. We solve (58) - (60) numerically (neglecting the equations for the ion concentrations, $(59)$ and $(60 \mathrm{~b})$ ), subject to the initial and boundary conditions (65) and (66), until a steady-state solution for the saturation is obtained. We repeat this procedure for 50 randomly generated networks, and compute the averaged solution.

\footnotetext{
${ }^{3}$ We impose a constant capillary pressure at the inlet nodes, rather than a constant saturation, in order to prevent flow reversal.
} 


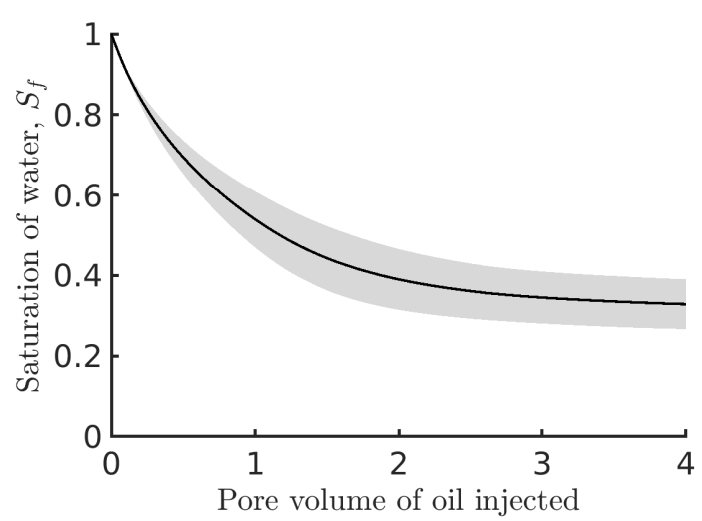

(a)

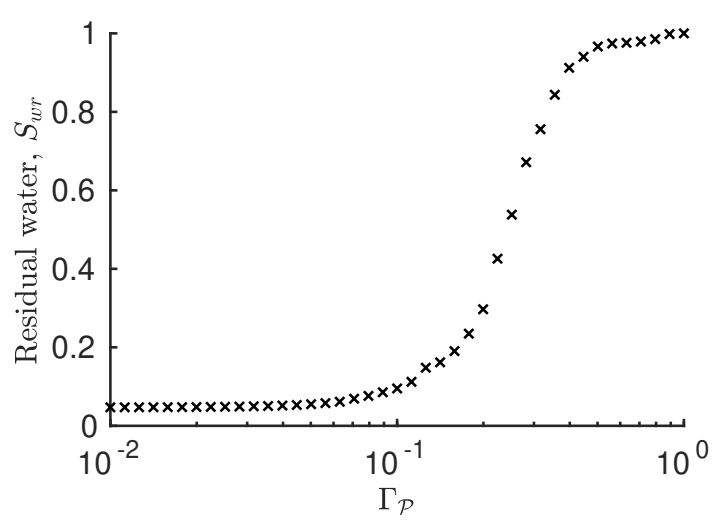

(b)

Figure 4: (a) Saturation of water against pore volume injected during the drainage stage, with $\Gamma_{\mathcal{P}}=0.2$, averaged over 50 realisations of the $30 \times 30$ network. The shaded region shows the standard deviation from the mean. (b) Residual water saturation, $S_{w r}$, as a function of $\Gamma_{\mathcal{P}}$

In Figure 4a, we plot the total volume fraction of water in the internal (non-boundary) nodes of the networks, $S_{f}=\mathbf{S}_{M} \cdot \mathbf{V}_{M} / \sum_{i=a+1}^{N-b} V_{i}$, against the volume of oil injected, which we measure as a fraction of the total 'pore volume' which we define to be the total volume of fluid space in the network, $\sum_{i} V_{i}$. We plot the solution averaged over the 50 instances, with the standard deviation from the mean shown by the shaded region. The networks are initially saturated with water, so that $S_{f}=1$, and the average volume fraction decreases to $S_{f}=0.33$, after 4 pore volumes of oil have been injected. As $t \rightarrow \infty$, the solution approaches a steady-state limit, $\mathbf{S}_{M} \rightarrow \mathbf{S}_{W}^{0}$, where the $W$ subscript denotes 'waterflood', which we will use as our initial condition for the saturation in the waterflood stage.

In Figure 4b, we plot the effective drainage phase capillary pressure-saturation curve, $S_{w r}$ versus $\Gamma_{\mathcal{P}}$, after 4 pore volumes of oil have been injected for a single example of a $30 \times 30$ network. We observe that the residual water saturation asymptotically approaches a non-zero value (0.05) as $\Gamma_{\mathcal{P}} \rightarrow 0$, (i.e. as the applied pressure increases). As $\Gamma_{\mathcal{P}}$ increases (i.e. the applied pressure decreases), the residual water increases until some threshold pressure is reached, at which point the water saturation remains equal to unity since the pressure in the oil phase is not sufficient to overcome the entry pressure of the inlet throats. Consequently, oil cannot invade the network. It is worth noting that the residual water saturation will depend on the order in which pores are invaded during drainage, which may also vary with $\Gamma_{\mathcal{P}}$.

\subsection{Waterflood stage}

Having determined an initial condition for the saturation, we need to define appropriate boundary conditions for the waterflood stage. We assume that the inlets of the network are connected to water, so that $S_{i}^{\text {in }}=1$ for $1 \leq i \leq a$, and we assume that the water phase is subject to a 'waterflood' pressure difference, $P_{W}$. We set our pressure scale to be $\mathcal{P}=P_{W}$, so that the dimensionless initial and boundary conditions for the pressure and saturation read

$$
\mathbf{S}_{I M}=\mathbf{S}_{W}^{0}, \quad \text { at } t=0,
$$




$$
P_{i}^{\text {in }}=1, \quad S_{i}^{\text {in }}=1, \quad \text { for } 1 \leq i \leq a,
$$

where we have used the fact that $P_{i}=p_{i}^{w}$ at the inlets, since $S_{i}=1$.

A typical pressure drop across a $10 \mu \mathrm{m}$ throat is $\Delta P \approx 0.045 \mathrm{~kg} \mathrm{~m}^{-1} \mathrm{~s}^{-2}$ (Wilmott et al., 2018). Hence, for our network comprising 30 throats in the direction of flow, we estimate that $\mathcal{P} \approx 1.35 \mathrm{~kg} \mathrm{~m}^{-1} \mathrm{~s}^{-2}$, and therefore $\Gamma_{\mathcal{P}} \approx 1.48 \times 10^{4}$, during the waterflood stage. However, by neglecting snap-off and contact angle hysteresis from our model, we find that the system is independent of $\Gamma_{\mathcal{P}}$ during waterflooding ${ }^{4}$ due to the cancellation of $\Gamma_{\mathcal{P}}$ between (44) and (47) when used in (43). Hence, for an arbitrarily prescribed inlet pressure, our model describes capillarydominated flow. This is consistent with our estimation for $\Gamma_{\mathcal{P}}$ (which corresponds to a capillary number of $C a=R^{t} / 30 L^{t} \Gamma_{\mathcal{P}}=O\left(10^{-7}\right)$ ), and is the result of our assumption that there are multiple interfaces which may be approximated by a well-mixed solution. If we were to include contact angle hysteresis or snap-off in our model this would no longer be the case.

It is worth noting that we prescribed $\mathcal{P}$ to be significantly larger in the drainage stage (see $\S 4.1$ ), than in the waterflood stage, in order for the pressure in the oil-filled inlet nodes to be large enough for the oil phase to invade the network. If the pressure was set lower, the capillary pressure wouldn't be large enough to overcome the entry pressure requirements of the inlet throats.

In order to capture both secondary and tertiary waterflooding, we suppose that the concentration of ions at the inlet is initially given by the high-salinity concentration, until a time, $T$, at which low salinity water is injected at concentration $c_{\text {low }}^{k+}$. Since the concentration variables in our model are scaled with the concentration in the high salinity regime, this reads

$$
c_{i n}^{k+}(t)= \begin{cases}1, & 0 \leq t \leq T, \\ c_{\text {low }}^{k+} / c_{\text {high }}^{k+}, & t>T .\end{cases}
$$

Setting $T=0$ corresponds to a secondary stage low-salinity waterflood, setting $T=\infty$ corresponds to a secondary stage high-salinity waterflood, and setting $T$ to be finite but non-zero corresponds to a secondary high-salinity waterflood, followed by a tertiary low-salinity waterflood. We consider the three cases given by $T=0, T=\infty$, and $T$ finite but greater than the time taken for the high-salinity waterflood to reach a steady state.

In Figure 5a, we show the percentage of oil recovered (which, by conservation of mass, is the same as the percentage increase in the volume of water in the network which we calculate from the change in the saturation), against the pore volume injected for the secondary stage high-salinity and low-salinity waterfloods. We plot the solutions averaged over the 50 instances, with the standard deviation from the mean shown by the shaded regions. We observe that, in both cases, the majority of the oil recovery is achieved after approximately 1 pore volume of water has been injected. The amount of oil recovered increases by approximately $4.9 \%$ (original oil-in-place), showing that our model, which incorporates the multicomponent ion exchange mechanism, reproduces the low salinity effect.

In Figure 5b, we show the percentage oil recovered as a function of volume injected for a secondary stage high-salinity waterflood followed by a tertiary stage low-salinity waterflood. We initiate the low-salinity waterflood after 3 pore volumes of high-salinity water have been injected. We observe that the amount of oil recovered increases after the low-salinity injection is initiated, showing that our model captures the low salinity effect during a tertiary recovery process. There is

\footnotetext{
${ }^{4}$ This is not the case during drainage due to the inlet boundary conditions.
} 


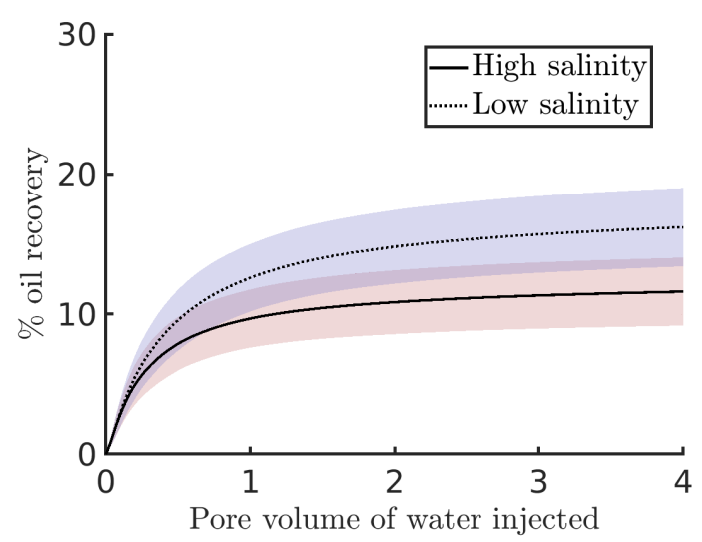

(a)

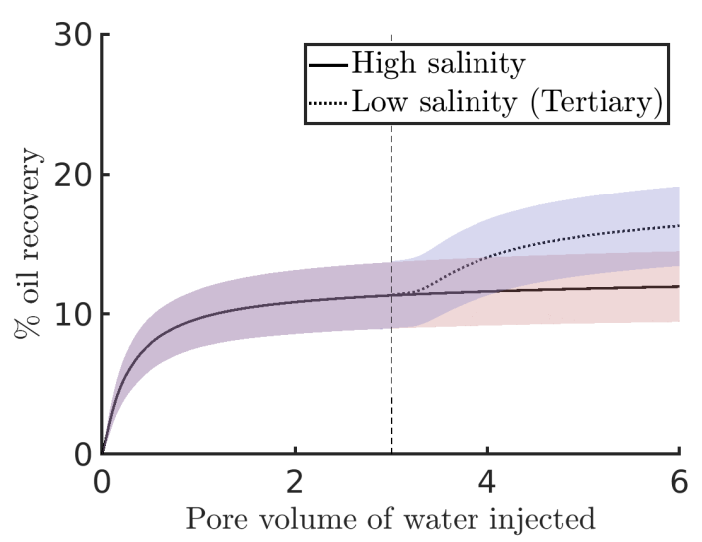

(b)

Figure 5: Graphs showing the percentage of oil recovered as a function of the pore volume of water injected averaged over 50 realisations of the $30 \times 30$ network, with the standard deviation from the mean shown by the shaded region, for: (a) a secondary-stage high salinity and low salinity injections; (b) a secondary-stage high salinity injection followed by a tertiary-stage low salinity injection. The vertical dashed line indicates the point at which the low salinity injection is initiated, and the dotted line shows the oil recovery for the high-salinity waterflood in which no tertiary low-salinity waterflood is performed.

a short delay between the start of the low-salinity injection and the point at which the additional recovery is observed. An additional $4.7 \%$ of the original oil-in-place is recovered during the tertiary low-salinity injection phase by comparison with high salinity injection alone.

In Figures $6 \mathrm{a}$ and $6 \mathrm{~b}$, we plot the residual oil distributions after a high salinity and low salinity waterflood have been performed, respectively, for a single network. We observe that, in both cases, the distribution is highly heterogeneous. There is less residual oil after a low salinity injection has been performed. However, the fluid distribution is similar in each case.

In Figure 7a, we show the residual oil saturation, $S_{\text {or }}$, against the oil-water viscosity ratio, $\mu^{*}$, after 3 pore volumes of water have been injected for both the low-salinity and high salinity waterflood cases. We observe that the residual oil saturation increases as $\mu^{*}$ increases in the high salinity case due to a decrease in the throat conductivities, as can be seen in (42). In the low salinity case, the residual oil initially increases with $\mu^{*}$ but decreases again for $\mu^{*} \gtrsim 6$ due to the low salinity effect. In Figure $7 \mathrm{~b}$, we plot the percentage recovery increase during a low salinity waterflood by comparison with a high salinity waterflood as a function of $\mu^{*}$. We observe that the percentage recovery increases approximately linearly as $\mu^{*}$ increases. This is due to the fact that the slip length increases proportionally with $\mu^{*}-1$, as seen in (46).

\section{Conclusions}

We have used our mathematical model for the salinity-dependent motion of an oil slug moving through a brine-filled capillary (Wilmott et al., 2018) to develop a two-phase network model for the effect of the multicomponent ionic exchange reactions on the oil recovery process. In our model, we assume that both the water and oil phases can be present in throats and pores at the same time, with the oil phase occupying the centres of the pores and forming slugs surrounded by thin 


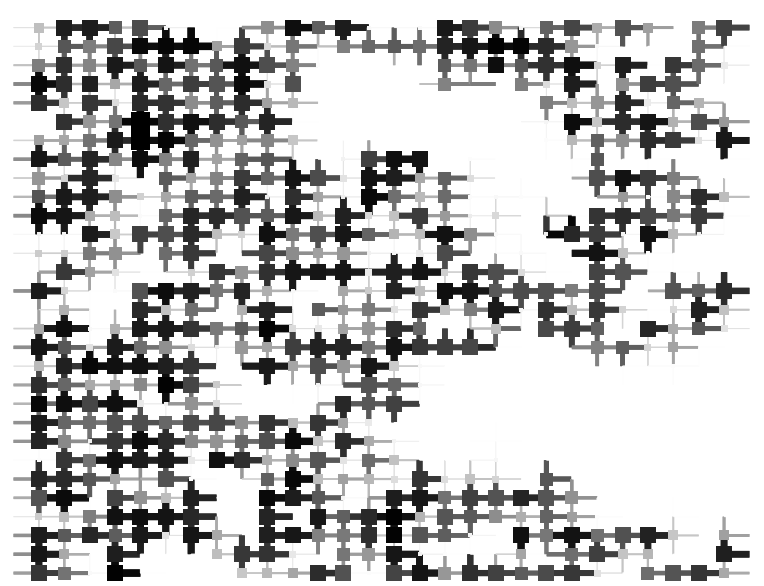

(a) High salinity residual oil

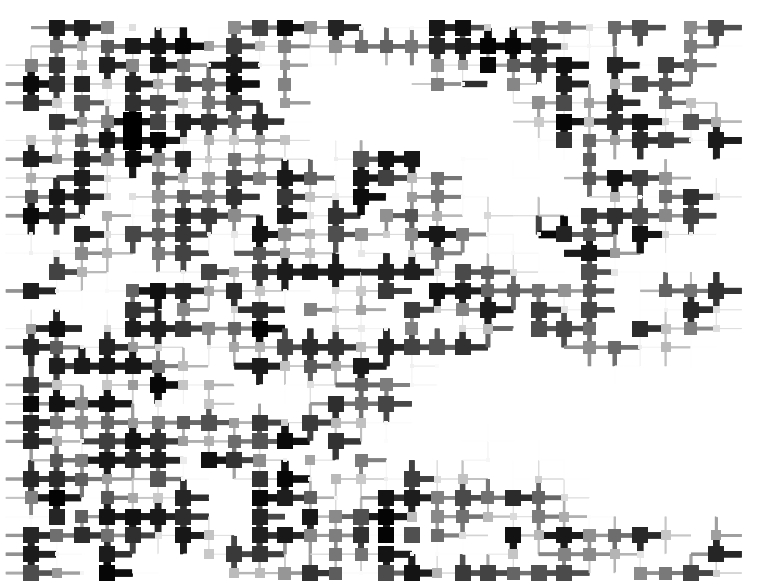

(b) Low salinity residual oil

Figure 6: Distributions of the residual oil after waterflooding for (a) a high salinity injection and (b) a low salinity injection. The thickness and intensity of the nodes and edges correspond to the amount of oil in the pores and throats, respectively.

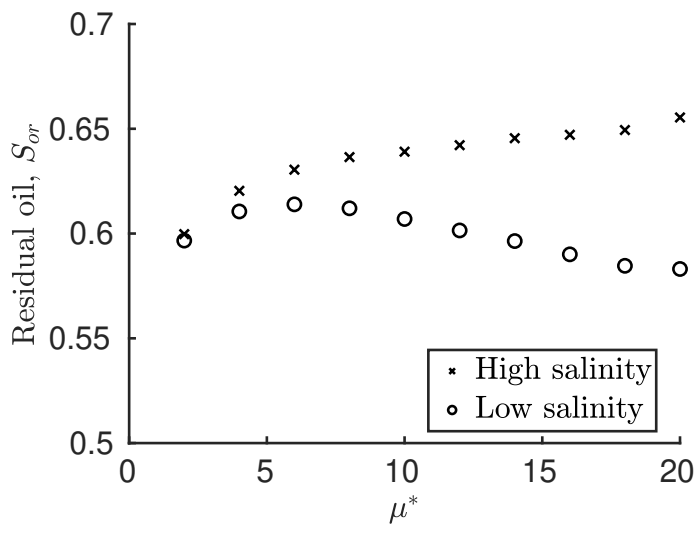

(a)

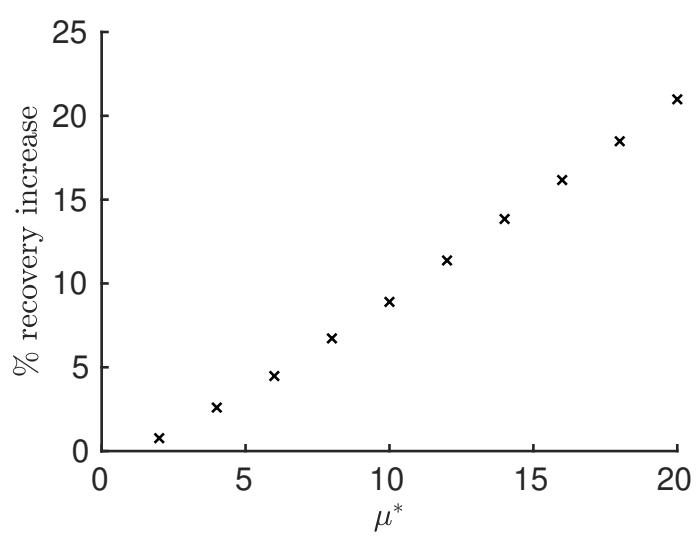

(b)

Figure 7: Plots showing: (a) residual oil saturation, $S_{\text {or }}$, against $\mu^{*}$ for the high-salinity and low-salinity secondary waterflood cases; (b) percentage oil recovery increase obtained during a low salinity waterflood relative to a high salinity waterflood as a function of $\mu^{*}$. 
films of water in the throats. For the case in which the throat geometry is uniform along its length, and the radii of curvature of the advancing and receding menisci of the oil slugs are the same, we found that the conductivity of the throat, given by (14), is independent of the number of oil slugs formed and depends only on the volume fraction of each phase within the throat. This enabled us to allow multiple oil slugs to form within a given throat in our model without having to track multiple interfaces, as is required in similar models such as those based on the Washburn equation.

In $\S 2$, we derived governing equations for the total flux and water saturation using conservation of mass for both the total fluid and for the water phase. We formulated the model in terms of the phaseweighted pressure in order to solve the system using a single set of pressure equations, rather than one for each phase as in the annular flow model discussed in $\S 1.1$, which reduces the computational effort required. We found that it may be appropriate to prescribe non-uniform boundary conditions for the phase-weighted pressure and saturation across the inlet or outlet nodes, in order to ensure that the pressure within each phase is uniform at the inlet and outlet. We included the effect of salinity in our model by assuming that there is a thin film of water separating the oil phase from the surface of the pore throat, with thickness determined by the salinity of the surrounding brine. We prescribed the thickness of this layer using the model for multicomponent ion exchange derived in Wilmott et al. (2018).

In $\S 3$, we non-dimensionalised our model and found that the system depends on seven dimensionless parameters; the oil-water viscosity ratio $\mu^{*}=\mu_{o} / \mu_{w}$, the ratio of the Debye length to a typical pore radius, $\epsilon_{2}=\left(\varepsilon_{w} k_{B} T / 2 c_{I}^{1+} q^{2}\right)^{1 / 2} / R^{t}$, the ratio of divalent ions to monovalent ions, $c^{*}=c_{I}^{2+} / c_{I}^{1+}$, the ratio of a typical throat radius to a typical pore radius, $R^{*}=R^{t} / R^{p}$, the ratio of the concentration of cation exchange sites on the oil surface to the concentration of cation exchange sites on the capillary surface, $s^{*}=s_{o}^{*} / s_{c}^{*}$, the ratio of the surface tension induced pressure to the electrostatic pressure within the thin water film, $\Gamma=2 \gamma \varepsilon_{w} R^{t} / L^{t^{2}} q^{2} s_{c}^{* 2}$, and the ratio of the surface tension induced pressure to the applied pressure, $\Gamma_{\mathcal{P}}=\gamma / R^{t} \mathcal{P}$. We then formulated our model using vector notation, and derived an implicit pressure explicit saturation numerical scheme to solve for the pressure $\mathbf{P}$, saturation $\mathbf{S}$, and ion concentrations, $\mathbf{c}^{k+}$.

In order to set up a reservoir with sensible distributions of oil and water, we first performed a 'drainage' stage in which oil invades a water-filled network by the action of capillary forces, simulating the initial formation of the oil reservoir. We then performed 'waterflood' simulations, in which the oil-filled steady state determined during the drainage stage is injected with water to remove oil until a steady state is obtained. We compared the results of a high-salinity injection with a low-salinity injection, and found that, while the initial rate of recovery was the same in both cases, the high-salinity recovery rate decreased before the low-salinity recovery rate, resulting in additional oil being produced during the low salinity injection. We also considered the case in which a high salinity injection is followed by a low salinity injection, simulating a tertiary oil recovery process. Again, we found that additional oil is produced during the low-salinity injection stage. In our simulations, we found that low salinity water produced $4.9 \%$ or $4.7 \%$ more oil (original oil-in-place) when injected as a secondary or tertiary process, respectively, by comparison with high-salinity injection alone. This is slightly less than observed in experimental studies, which find that the amount of additional oil recovered after low salinity injection is typically between $5 \%$ and 10\% original oil-in-place (Rezaeidoust et al., 2011; Ligthelm et al., 2009; Tang and Morrow, 1999). This is likely due to real pore spaces having greater connectivity in three-dimensional space, as well as additional effects such as snap-off and contact angle hysteresis being neglected from our model. If we were to include the effects of snap-off in our model, we would likely see large regions of trapped oil since the snap-off mechanism enables the oil to become disconnected in the middle 
of the connected oil phase as opposed to our current model in which oil becomes trapped primarily due to downstream entry pressure thresholds. Additional work is required to incorporate these effects into a comprehensive model.

In summary, our model is able to qualitatively reproduce the low salinity effect observed experimentally, as both a secondary-stage process and as a tertiary-stage process. While the focus of this paper is on the multicomponent ionic exchange mechanism, the network modelling approach we have presented here provides a framework for studying alternative low salinity processes. This enables the study of these mechanisms on a much larger length scale than was previously possible. Additional work will be required to upscale the network model to a length scale of practical interest, such as a core plug, as well as to model the system in three dimensions. One possible homogenisation approach is to apply the discrete method of multiple scales to our network model in order to derive a continuum model for the effect of salinity on the oil recovery process which applies on the macroscopic scale (Chapman and Shabala, 2017). Such an approach will be crucial in order to accurately determine the dominant causal mechanism, and to quantitatively relate the parameters which govern the microscale physics to the macroscale recovery process.

\section{Acknowledgements}

This work was supported by the Engineering and Physical Sciences Research Council and BP in the form of a CASE conversion, grant number EP/M50659X/1. We would like to thank Bilal Rashid, Tim Totton, John Couves, and Pete Salino for their support throughout the project.

\section{References}

M. S. Al-Gharbi and M. J. Blunt. Dynamic network modeling of two-phase drainage in porous media. Physical Review E, 71(1):016308, 2005.

V. Alvarado, G. Garcia-Olvera, P. Hoyer, T. E. Lehmann, et al. Impact of polar components on crude oil-water interfacial film formation: A mechanisms for low-salinity waterflooding. In SPE Annual Technical Conference and Exhibition. Society of Petroleum Engineers, 2014.

T. Austad, A. Rezaeidoust, and T. Puntervold. Chemical mechanism of low salinity water flooding in sandstone reservoirs. In SPE improved oil recovery symposium, page 129767. Society of Petroleum Engineers, 2010.

S. Berg, A. Cense, E. Jansen, and K. Bakker. Direct experimental evidence of wettability modification by low salinity. Petrophysics, 51(5):314-322, 2010.

J. S. Buckley. Mechanisms and consequences of wettability alteration by crude oils. PhD thesis, Department of Petroleum Engineering, Heriot-Watt University, 1996.

J. S. Buckley, K. Takamura, N. R. Morrow, et al. Influence of electrical surface charges on the wetting properties of crude oils. SPE Reservoir Engineering, 4(3):332-340, 1989.

S. J. Chapman and A. Shabala. Effective transport properties of lattices. SIAM Journal on Applied Mathematics, 77(5):1631-1652, 2017.

H. K. Dahle and M. A. Celia. A dynamic network model for two-phase immiscible flow. Computational Geosciences, 3(1):1-22, 1999. 
C. Drummond and J. Israelachvili. Fundamental studies of crude oil-surface water interactions and its relationship to reservoir wettability. Journal of Petroleum Science and Engineering, 45(1): 61-81, 2004.

P. Fletcher and G. Sposito. Chemical modeling of clay/electrolyte interactions of montmorillonite. Clay Minerals, 24(2):375-391, 1989.

P. Fournier, E. H. Oelkers, R. Gout, and G. Pokrovski. Experimental determination of aqueous sodium-acetate dissociation constants at temperatures from 20 to 240 C. Chemical Geology, 151 (1):69-84, 1998.

N. J. Hadia, T. Hansen, M. T. Tweheyo, and O. Torsæter. Influence of crude oil components on recovery by high and low salinity waterflooding. Energy $\mathscr{E}$ Fuels, 26(7):4328-4335, 2012.

R. J. Held and M. A. Celia. Modeling support of functional relationships between capillary pressure, saturation, interfacial area and common lines. Advances in Water Resources, 24(3-4):325-343, 2001.

P. Jadhunandan. Effects of brine composition, crude oil, and aging conditions on wettability and oil recovery. PhD thesis, Department of Petroleum Engineering, New Mexico Institute of Mining \& Technology, 1990.

P. Jadhunandan and N. R. Morrow. Spontaneous imbibition of water by crude oil/brine/rock systems. In Situ;(United States), 15(4):319-345, 1991.

P. Jadhunandan and N. R. Morrow. Effect of wettability on waterflood recovery for crudeoil/brine/rock systems. SPE Reservoir Engineering, 10(1):40-46, 1995.

V. Joekar-Niasar and S. M. Hassanizadeh. Analysis of fundamentals of two-phase flow in porous media using dynamic pore-network models: A review. Critical reviews in environmental science and technology, 42(18):1895-1976, 2012.

V. Joekar-Niasar, S. M. Hassanizadeh, and A. Leijnse. Insights into the relationships among capillary pressure, saturation, interfacial area and relative permeability using pore-network modeling. Transport in Porous Media, 74(2):201-219, 2008.

V. Joekar-Niasar, S. M. Hassanizadeh, and H. K. Dahle. Non-equilibrium effects in capillarity and interfacial area in two-phase flow: dynamic pore-network modelling. Journal of Fluid Mechanics, 655:38-71, 2010.

N. R. Joseph. The dissociation constants of organic calcium complexes. Journal of Biological Chemistry, 164(2):529-541, 1946.

H. A. Knudsen, E. Aker, and A. Hansen. Bulk flow regimes and fractional flow in 2D porous media by numerical simulations. Transport in Porous Media, 47(1):99-121, 2002.

J. Koplik and T. J. Lasseter. One-and two-phase flow in network models of porous media. Chemical Engineering Communications, 26(4-6):285-295, 1984.

A. Lager, K. Webb, C. Black, M. Singleton, and K. Sorbie. Low salinity oil recovery-an experimental investigation. Petrophysics, 49(1):28-35, 2008. 
S. Lee, K. Webb, I. Collins, A. Lager, S. Clarke, M. O'Sullivan, A. Routh, and X. Wang. Low salinity oil recovery: Increasing understanding of the underlying mechanisms. In SPE improved oil recovery symposium, page 129722. Society of Petroleum Engineers, 2010.

W. C. M. Lewis. The electric charge at an oil-water interface. Transactions of the Faraday Society, 33:708-713, 1937.

S. Li and R. Xu. Electrical double layers' interaction between oppositely charged particles as related to surface charge density and ionic strength. Colloids and Surfaces A: Physicochemical and Engineering Aspects, 326(3):157-161, 2008.

D. J. Ligthelm, J. Gronsveld, J. Hofman, N. Brussee, F. Marcelis, and H. van der Linde. Novel waterflooding strategy by manipulation of injection brine composition. In EUROPEC/EAGE Conference and Exhibition, page 119835. Society of Petroleum Engineers, 2009.

C. G. Malmberg and A. A. Maryott. Dielectric constant of water from 0 to 100 C. J Res Nat Bureau Stand, 56:1-8, 1956.

M. Moradi, V. Alvarado, and S. Huzurbazar. Effect of salinity on water-in-crude oil emulsion: evaluation through drop-size distribution proxy. Energy \& fuels, 25(1):260-268, 2010.

N. R. Morrow. Wettability and its effect on oil recovery. Journal of Petroleum Technology, 42(12): $1-476,1990$.

N. A. Mortensen, F. Okkels, and H. Bruus. Reexamination of Hagen-Poiseuille flow: Shape dependence of the hydraulic resistance in microchannels. Physical Review E, 71(5):057301, 2005.

P. Nelson. Pore-throat sizes in sandstones, tight sandstones, and shales. AAPG bulletin, 93(3): 329-340, 2009.

A. Rezaeidoust, T. Puntervold, and T. Austad. Chemical verification of the EOR mechanism by using low saline/smart water in sandstone. Energy \& Fuels, 25(5):2151-2162, 2011.

Q. Sheng and K. Thompson. A unified pore-network algorithm for dynamic two-phase flow. Advances in Water Resources, 95:92-108, 2016.

G. Q. Tang and N. R. Morrow. Influence of brine composition and fines migration on crude oil/brine/rock interactions and oil recovery. Journal of Petroleum Science and Engineering, 24 (2):99-111, 1999.

K. E. Thompson. Pore-scale modeling of fluid transport in disordered fibrous materials. AIChE journal, 48(7):1369-1389, 2002.

$\mathrm{X}$. Wang and V. Alvarado. Effects of aqueous-phase salinity on water-in-crude oil emulsion stability. Journal of Dispersion Science and Technology, 33(2):165-170, 2012.

E. W. Washburn. The dynamics of capillary flow. Physical review, 17(3):273-283, 1921.

Z. M. Wilmott, C. J. W. Breward, and S. J. Chapman. The effect of ions on the motion of an oil slug through a charged capillary. Journal of Fluid Mechanics, 841:310-350, 2018.

Z. M. Wilmott, C. J. W. Breward, and S. J. Chapman. Modelling low-salinity oil recovery mechanisms using an ion dissociation model. Transport in Porous Media, 127(3):685-709, 2019. 


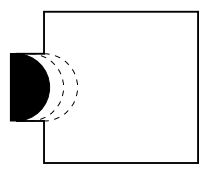

I

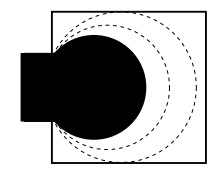

II

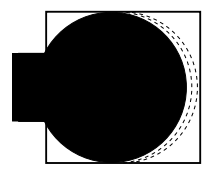

III

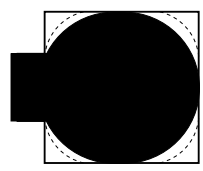

IV

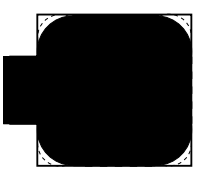

V

Figure A.8: Schematic diagram showing the five stages of the pore-filling process.

\section{Appendix A. Capillary-pressure-saturation relationship}

The capillary pressure between the two phases in a pore depends on both the saturation and the geometry of the pore. As a simple example, we consider a square pore in two dimensions as shown in Figure A.8. We suppose that the pore has sides of length $2 R^{p}$, and that the radius of the throat through which the invading phase enters is $R^{t}$. For simplicity, we neglect additional throats which may be connected to the pore. We non-dimensionalise by scaling the lengths with $2 R^{p}$, so that the pore has dimensionless side-length of unity.

Following the approach of Joekar-Niasar et al. (2010), we separate the pore-filling process into five stages, shown in Figure A.8. These are

- Stage I: The invading phase first enters the pore, and the curvature of the interface is determined by the radius of the entry throat.

- Stage II: The invading phase is pinned at the corners of the entry throat, occupying a truncated circular region which expands within the pore until the interface reaches the edges of the pore.

- Stage III: The interface of the invading fluid advances through the pore until it reaches the side opposite the entry throat.

- Stage IV: The interface remains pinned at the entry throat corners, and the curvature of the interface increases until it lies tangentially to the pore walls.

- Stage V: The interface expands into the corners of the pore, with the curvature of the interface increasing in order to remain tangential to the pore walls.

During stage I, when the invading phase first enters the pore, the curvature of the interface is determined by the radius of the pore throat through which the fluid enters, and the capillary pressure is given by

$$
P^{c}=4 \Gamma_{\mathcal{P}}
$$

where $\Gamma_{\mathcal{P}}=\gamma / R^{t} \mathcal{P}$ is the ratio of the surface tension-induced pressure to the applied pressure, $\mathcal{P}$. The capillary pressure remains at this value until the invading fluid reaches the corners of the entrance to the pore, forming a semi-circular cap. By subtracting the area of the semi-circular oil region from the area of the pore, we find that the water saturation at the end of stage I is given by $S=1-\frac{\pi}{8} R^{* 2}$, where $R^{*}=R^{t} / R^{p}$ is the ratio of the throat radius to the pore radius. Hence, for $1-\frac{\pi}{8} R^{* 2}<S \leq 1$, the capillary pressure is given by (A.1).

During stage II, the invading phase forms a truncated circle, with the edge of the circle being pinned to the two corners at the entrance of the pore. By computing the area outside the truncated 
circle, for a given radius $r$, we find that the water saturation during this stage is given by

$$
S=1-r^{2}\left(\pi-\arcsin \left(\frac{R^{*}}{2 r}\right)+\frac{R^{*}}{2 r} \sqrt{1-\left(\frac{R^{*}}{2 r}\right)^{2}}\right) .
$$

The capillary-pressure-saturation relationship may then be found implicitly from $P^{c}=2 R^{*} \Gamma_{\mathcal{P}} / r$. As the saturation decreases, $r$ increases until $r=1 / 2$, at which point the invading phase touches the upper and lower pore walls and the saturation is given by

$$
S=1-\frac{1}{4}\left(\pi-\arcsin R^{*}+R^{*} \sqrt{1-R^{* 2}}\right) .
$$

During stage III, the advancing front moves forward with constant curvature, and the capillary pressure remains constant, given by $P^{c}=4 R^{*} \Gamma_{\mathcal{P}}$, until the interface reaches the far pore wall. We compute the saturation at which the interface touches the far pore wall by computing the area of the water region, which reads

$$
S=1-\frac{1}{4}\left(2+\pi-\arcsin R^{*}+\left(R^{*}-2\right) \sqrt{1-R^{* 2}}\right) .
$$

During stage IV, the invading phase is pinned to the corners at the entrance of the pore, and the saturation is given by

$$
S=r^{2}\left(2-\pi+\arcsin \left(\frac{2 r+R^{*}-1}{2 r}\right)+\frac{2 r-R^{*}+1}{2 r} \sqrt{1-\left(\frac{2 r+R^{*}-1}{2 r}\right)^{2}}\right),
$$

where $r$ is the radius of curvature of the invading fluid in the corners of the pore. The capillary pressure is determined implicitly using $P^{c}=2 R^{*} \Gamma_{\mathcal{P}} / r$, and increases until the fluid interface lies tangentially to the pore wall, at which point $r=\left(1-R^{*}\right) / 2$.

In the final stage, the invading phase is squeezed into the corners of the pore, and the saturation is found by computing the area outside the quarter-circular regions with radius $r$ in each of the four corners of the pore, which reads

$$
S=(4-\pi) r^{2}
$$

Hence, by substituting $P^{c}=2 R^{*} \Gamma_{\mathcal{P}} / r$ and rearranging, we find that the capillary pressure is given by

$$
P^{c}=2 R^{*} \Gamma_{\mathcal{P}} \sqrt{\frac{4-\pi}{S}}
$$

In Figure A.9 we plot the capillary pressure, given by (A.1) - (A.7), against the saturation, for $R^{*}=0.5$ and $\Gamma_{\mathcal{P}}=0.2$, indicating each stage of the invasion process using dashed red lines. During stages I and III, the capillary pressure is constant as the saturation varies, during stage II, the capillary pressure decreases as the saturation decreases, and, during stages IV and V, the capillary pressure increases as the saturation decreases, asymptotically approaching a singularity at $S=0$. Since the capillary pressure must be greater than the throat entry pressure in order for the invading phase to enter the throat in the first place, we find that, when the invading fluid first enters the pore, the pore rapidly fills to the point during the fourth or fifth stage of the pore-filling process at which the capillary pressure is equal to the entry pressure of the throat. Hence, the pore fills 


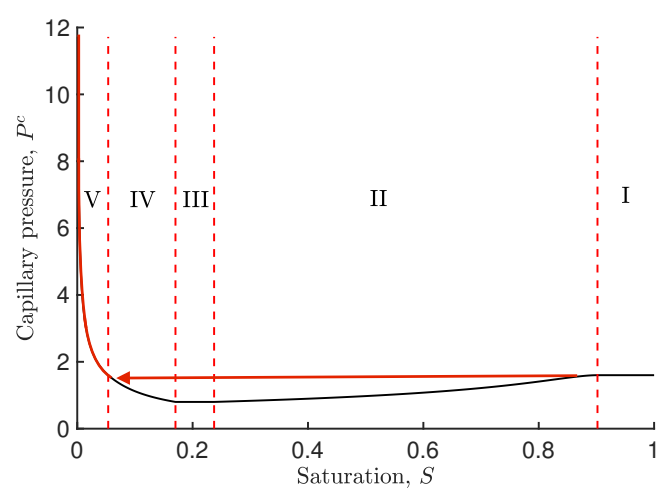

Figure A.9: Graph showing the dimensionless capillary pressure, given by (A.1) - (A.7), as a function of the water saturation with $R^{*}=0.5$ and $\Gamma_{\mathcal{P}}=0.2$. The boundaries of the five stages of the invasion process are shown with dashed red lines.

instantaneously, so we can simplify the capillary-pressure-saturation function by neglecting the first three stages of the pore filling process during invasion. We also show how the saturation 'jumps' as the invading fluid enters the pore: we highlight in red the section of the capillary-pressure-saturation function which may be inverted to determine the saturation during invasion.

To simplify the computations, we approximate the fourth and fifth stages of the pore filling process using a power-law model. We suppose that the capillary pressure is of the form $2 R^{*} \Gamma_{\mathcal{P}} / a S^{b}$ in regions IV and V, rearrange to write $\log a+b \log S=\log \left(2 R^{*} \Gamma_{\mathcal{P}} / P^{c}\right)$, and fit $\log a$ and $b$ to the solution for $R^{*}=0.3$ in regions IV and $\mathrm{V}$ using a least-squares algorithm, to find that $a \approx 1.1 \approx 1 / \sqrt{4-\pi}$, and $b \approx 0.5$. We therefore define

$$
P_{F}^{c}:=2 R^{*} \Gamma_{\mathcal{P}} \sqrt{\frac{4-\pi}{S}},
$$

to be our 'fitted' capillary pressure curve, valid in regions IV and V, noting that this is simply the extension of (A.7) into region IV. In Figure A.10, we plot (A.8) against the full capillary pressure model, (A.1) - (A.7), for $R^{*}=0.1,0.3,0.5$. We see that $R^{*}$ has little impact on the solution in stages IV and $\mathrm{V}$, and that the power-law model is in good agreement with the solutions for all values of $R^{*}$ in these latter stages of the invasion process. This is important, since $R^{*} \Gamma_{\mathcal{P}}=\gamma / R^{p} \mathcal{P}$ is independent of the throat radius and therefore our fitted capillary pressure curve, given by (A.8), depends only on the size of the pore. Hence, we can use a single capillary-pressure-saturation relationship for each pore in our network during the drainage stage, regardless of the throat through which the invading phase enters.

During a waterflood process, the pore is initially oil-filled. Hence, the saturation is low and the capillary pressure is determined by the region highlighted in red in Figure A.9. As water is injected, oil is expelled from the pore through an exit throat, and the water saturation increases. The capillary pressure decreases until it reaches the entry pressure of the exit throat. At this point, oil cannot exit the pore, and therefore the saturation cannot increase further. Hence, the pore remains filled according to stages IV and V of the filling process, and therefore we can use (A.8) to approximate the capillary-pressure-saturation relationship during waterflooding, as well as during drainage. 


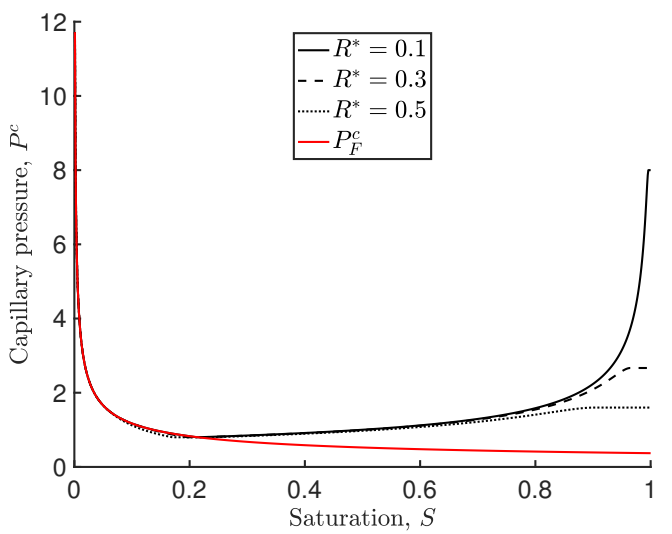

Figure A.10: Plots of the capillary pressure as a function of the saturation, given by (A.1) - (A.7), with $R^{*}=$ $0.1,0.3,0.5$ and $\Gamma_{\mathcal{P}}=0.2$, compared with the fitted solution, $P_{F}^{c}$, given by (A.8). 